\title{
Finding all flux vacua in an explicit example
}

\section{Danny Martínez-Pedrera, ${ }^{a}$ Dhagash Mehta, ${ }^{b}$ Markus Rummel ${ }^{a}$ and Alexander Westphal ${ }^{c}$}

\author{
${ }^{a}$ II. Institut für Theoretische Physik der Universität Hamburg, \\ D-22761 Hamburg, Germany \\ ${ }^{b}$ Department of Physics, Syracuse University, \\ Syracuse, NY 13244, U.S.A. \\ ${ }^{c}$ Deutsches Elektronen-Synchrotron DESY, Theory Group, \\ D-22603 Hamburg, Germany \\ E-mail: dbmehta@syr.edu, markus.rummel@desy.de, \\ alexander. westphal@desy.de
}

ABSTRACT: We explicitly construct all supersymmetric flux vacua of a particular CalabiYau compactification of type IIB string theory for a small number of flux carrying cycles and a given D3-brane tadpole. The analysis is performed in the large complex structure region by using the polynomial homotopy continuation method, which allows to find all stationary points of the polynomial equations that characterize the supersymmetric vacuum solutions. The number of vacua as a function of the D3 tadpole is in agreement with statistical studies in the literature. We calculate the available tuning of the cosmological constant from fluxes and extrapolate to scenarios with a larger number of flux carrying cycles. We also verify the range of scales for the moduli and gravitino masses recently found for a single explicit flux choice giving a Kähler uplifted de Sitter vacuum in the same construction.

KEYWORDS: Flux compactifications, dS vacua in string theory, Superstring Vacua

ArXiv EPRINT: 1212.4530 


\section{Contents}

1 Introduction 1

2 The complex structure of $\mathbb{C P}_{11169}^{4}[18] \quad 4$

2.1 Effective reduction of the moduli space 6

3 The polynomial homotopy continuation method 9

3.1 The algorithm $\quad 9$

$\begin{array}{ll}3.2 \text { The scan } & 11\end{array}$

$\begin{array}{lll}3.3 & \text { Distribution of parameters } & 12\end{array}$

$\begin{array}{lll}3.4 & \text { De Sitter vacua via Kähler uplifting } & 17\end{array}$

4 The minimal flux method $\quad 19$

$\begin{array}{ll}4.1 \text { The algorithm } & 19\end{array}$

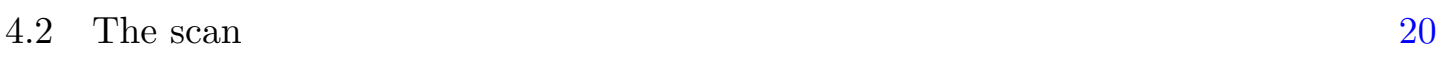

4.3 Results 21

5 Conclusions $\quad 23$

\section{Introduction}

The question of the vacuum states of string theory has been an outstanding problem in the field since its founding days. Those vacua which could describe our world are of particular interest. Apart from a standard model sector including the corresponding gauge groups and matter in the right representations of these gauge groups, such vacua should have a positive vacuum energy that is extremely small in terms of the natural scale of gravity, the Planck/string scale [1-3]. In addition, one may wish for 4D $\mathcal{N}=1$ supersymmetry for its power to keep control over quantum corrections to the theory above the supersymmetry breaking/electroweak scale. There has been remarkable progress in the construction of such de Sitter (dS) vacua with stabilized geometric moduli in the past years [4-6]. The moduli are 4D massless scalar fields parameterizing the geometric deformation modes of the compact six-dimensional spaces all viable Kaluza-Klein type vacua of string theory need to describe our effectively four-dimensional reality.

A corner of the string theory landscape where moduli stabilization can be addressed very explicitly is type IIB compactified on orientifolded Calabi-Yau threefolds. The fourdimensional effective action of the geometric moduli is given by an $\mathcal{N}=1$ theory of a set of chiral multiplets consisting of the axio-dilaton, $h^{1,1}$ Kähler moduli, and $h^{2,1}$ complex structure moduli [7]. Recent years saw a progress for the stabilization of the dilaton and the complex structure moduli from the use of quantized fluxes of three form field strength 
of the Ramond-Ramond and Neveu-Schwarz sector of type IIB string theory [5]. The flux stabilization procedure operates supersymmetrically at a high scale. The Kähler moduli are flat directions at tree level, i.e. they do not obtain a scalar potential due to the no-scale structure of type IIB compactified on Calabi-Yau [8, 9]. We can use the breaking of this no-scale structure by non-perturbative [6] and perturbative $[10,11]$ effects to stabilize the Kähler moduli at a parametrically lower scale than the complex structure moduli. This stabilization produces an AdS vacuum with unbroken supersymmetry [6], an AdS vacuum with spontaneously broken supersymmetry with an exponentially large volume $[12,13]$ or directly in a dS vacuum with spontaneously broken supersymmetry [14, 15].

If the stabilization of the geometric moduli does not directly lead to a dS vacuum, an additional uplifting sector has to be added. The uplifting may arise for instance by anti D3 branes [6], D-terms [16-18], F-terms from matter fields [19], metastable vacua in gauge theories [20] or dilaton dependent non-perturbative effects [21]. Concerning the smallness of the cosmological constant, statistical arguments show that due to the enormous amount of possible flux configurations there is an exponential abundance of isolated potential dS vacua [22-24]. For a sufficiently large number of complex structure moduli $h^{2,1}$ (typically $\mathcal{O}(100))$ the number of flux vacua and, in turn, also the number of dS vacua scaling like $e^{\mathcal{O}(1) h^{2,1}}$ is large enough to produce vacuum energies with average spacing $\lesssim 10^{-120}$. This enables the flux vacuum landscape in string theory in principle to accommodate the observed vacuum energy of our Universe.

In this paper, we study the flux vacua of a particular Calabi-Yau: the degree 18 hypersurface in a 4 complex dimensional projective space, i.e. $X_{3} \equiv \mathbb{C P}_{11169}^{4}[18]$ in the large complex structure limit. This manifold is the standard working example of both the large volume scenario (LVS) [12, 13] and the Kähler uplifting scenario [14, 15] and its geometric properties have been worked out in great detail in [25]. It has $h^{1,1}=2$ Kähler moduli and $h^{2,1}=272$ complex structure moduli. We switch on flux along six three-cycles that correspond to two complex structure moduli that are invariant under a certain discrete symmetry that can be used to construct the mirror manifold [26]. For this purpose we review a known argument that a supersymmetric vacuum in these two complex structure moduli corresponds to a supersymmetric vacuum of all 272 complex structure moduli [27, 28].

For an explicit construction of the flux vacua we use the fact that the prepotential $\mathcal{G}$ of the two complex structure moduli space has been worked out in [25] in the large complex structure limit. We apply two computational methods to find flux vacua on this manifold:

- The polynomial homotopy continuation method [29] allows us to find all stationary points of the polynomial equations that characterize the supersymmetric vacuum solutions. The fluxes $f_{i} \in \mathbb{Z}$ appear as parameters in these equations and are restricted by the D3 tadpole $L$ which depends on the chosen brane and gauge flux configuration imposed on the manifold. Since the restriction is of the form $\sum f_{i}^{2} \leq L$ this method allows us to explicitly construct for the first time all flux vacua in the large complex structure limit that are consistent with a given D3 tadpole $L$ by applying the polynomial homotopy continuation method at each point in flux parameter space. This method has the attractive feature to be highly parallelizable. 
- The minimal flux method [28] finds flux parameters that are consistent with a given D3 tadpole $L$ for a given set of vacuum expectation values (VEVs) of the complex structure moduli. Hence it is in a sense complementary to the polynomial homotopy continuation method where the role of parameters and solutions is exchanged with respect to this method. However, it is not possible to find all flux vacua for a given tadpole $L$ with this method.

The obtained solution space of flux vacua is analyzed for several physically interesting properties. For the polynomial homotopy continuation method, we find that for the $\sim 50,000$ flux choices contained in our maximum D3-brane tadpole $L=34$ of our scan there are $\sim 20,000$ solutions in the large complex structure limit. We find a preference of strongly coupled vacua $g_{s} \gtrsim 1$ and preference for values of $\mathcal{O}\left(10^{1}-10^{3}\right)$ for the flux superpotential $W_{0}$. The number of vacua is

$$
N_{\mathrm{vac}} \simeq(0.50 \pm 0.04) L^{2.94 \pm 0.03}
$$

compared to $\sim 0.03 L^{3}$ expected form statistical analysis $[24,30]$. The gravitino mass is typically $m_{3 / 2}^{2}=\mathcal{O}\left(10^{-3}\right) \cdot\left(\frac{100}{\mathcal{V}}\right)^{2} M_{\mathrm{P}}^{2}$ and the masses of the complex structure moduli and the dilaton scale like $\mathcal{O}\left(10^{-3}-10^{2}\right) \cdot\left(\frac{100}{\mathcal{V}}\right)^{2} M_{\mathrm{P}}^{2}$, where $\mathcal{V}$ is the Volume of $X_{3}$ in string units.

The average spacing of the flux superpotential in our solution set can be used to estimate the available fine-tuning $\Delta \Lambda / \Lambda$ of the cosmological constant $\Lambda$ as

$$
\frac{\Delta \Lambda}{\Lambda} \simeq(6.0 \pm 0.3) L^{-(0.95 \pm 0.005)\left(h_{\mathrm{eff}}^{2,1}+1\right)}
$$

where $h_{\text {eff }}^{2,1}$ is the number of complex structure moduli with non-zero flux on the corresponding three-cycles. Eq. (1.2) is obtained as a fit for $L \leq 34$ and $h_{\text {eff }}^{2,1}=2$. It can be used to estimate the available fine-tuning of the cosmological constant to for instance $\Delta \Lambda / \Lambda \sim 10^{-102}$ for $L=500$ and $h_{\text {eff }}^{2,1}=40$. These are typical values for Calabi-Yau manifolds that are hypersurfaces in toric varieties with D7 branes and O7 planes introduced to stabilize the Kähler moduli and break supersymmetry. The explicit brane and gauge flux construction in [15] allows us to answer the question how many of theses supersymmetric flux vacua allow an uplift to dS via Kähler uplifting. Depending on the available values for the one-loop determinant from gaugino condensation used to stabilize the Kähler moduli in this setup, we find that for a fraction of about $10^{-4}$ of all flux vacua up to a given D3-brane tadpole this mechanism can be applied to obtain a dS vacuum.

For the minimal flux method, we find $\sim 1000$ flux vacua with $L<500$ out of $\sim 10^{7}$ parameter points of our scan. This method allows us to control the region in $W_{0}$ and moduli space where we are intending to find flux vacua. Hence, we more easily access the regions of weak string coupling and the large complex structure limit compared to the polynomial homotopy continuation method. For this much smaller set of flux vacua constructed with the minimal flux method, the fraction of Kähler uplifted dS minima is about 10\%. This is a considerably higher fraction of vacua compared to the polynomial homotopy continuation method which is due to the fact that the minimal flux method naturally finds values for $W_{0}$ in a region where Kähler uplifting is applicable. 
Our results are complementary to statistical analysis by $[24,30] .{ }^{1}$ The uniform distribution of physical quantities as for instance the gravitino mass and the vacuum energy density in the landscape has recently been questioned in general [34-36] and in the context of Kähler uplifting [37-39]. Hence, our results present an important check of the general results found in $[24,30]$ on a very realistic examples $X_{3}$, especially since we are able to construct the complete solution space of flux vacua for a given tadpole $L$.

In section 2 , we review the effective $4 \mathrm{~d}, \mathcal{N}=1$ description of the complex structure moduli and the dilaton as well as the reduction of the full moduli space to two complex structure moduli. The scans for flux vacua with the polynomial homotopy continuation method and the minimal flux method are presented in section 3 respectively section 4 . We conclude in section 5 .

\section{The complex structure of $\mathbb{C P}_{11169}^{4}[18]$}

The effective $4 \mathrm{~d}, \mathcal{N}=1$ description of the moduli space of the $h^{2,1}=272$ complex structure moduli of $X_{3}$ is given by the Kähler potential $K$ and superpotential $W_{0}$ of the theory. ${ }^{2}$ We choose a symplectic basis $\left\{A_{a}, B^{b}\right\}$ for the $b_{3}=2 h^{2,1}+2$ three-cycles

$$
\int_{A_{a}} \alpha^{b}=\int_{X_{3}} \alpha^{b} \wedge \beta_{a}=\delta_{a}^{b}, \quad \int_{B^{b}} \beta_{a}=\int_{X_{3}} \beta_{a} \wedge \alpha^{b}=-\delta_{a}^{b},
$$

where $\left\{\alpha_{b}, \beta^{a}\right\}$ are the Poincaré dual cohomology elements to the three-cycles and $a, b=$ $0, \ldots, h^{2,1}$.

Having chosen a symplectic basis for the three-cycles, this defines a choice of coordinates $\omega_{a}$ on complex structure moduli space via the period integrals over the holomorphic three-form $\Omega$ via

$$
\omega_{a}=\int_{A_{a}} \Omega, \quad \mathcal{G}_{b}=\int_{B^{b}} \Omega .
$$

Note, that there are $h^{2,1}+1$ coordinates $\omega_{0}, \ldots, \omega_{h^{2,1}}$ even though there are only $h^{2,1}$ complex structure moduli. This is because $\omega_{0}$ refers to the normalization of the holomorphic three-form $\Omega$. The complex structure moduli can be defined via $U_{a} \equiv \nu_{a}+i u_{a}=\omega_{a} / \omega_{0}$ for $a=1, \ldots, h^{2,1}$. The period vector $\Pi\left(\omega_{a}\right)=\left(\mathcal{G}_{b}, \omega_{a}\right)$ is inherited from a holomorphic function $\mathcal{G}\left(\omega_{a}\right)$ of degree two in the $\omega_{a}$ known as the prepotential via $\mathcal{G}_{b}=\partial_{b} \mathcal{G}$ of the underlying $\mathcal{N}=2$ Calabi-Yau compactification.

The Kähler potential of the complex structure moduli $U_{a}$ and the dilaton $\tau=\sigma+i s$ can then be written as

$$
\begin{aligned}
K_{\mathrm{cS}} & =-\log \left(-i \int_{X_{3}} \Omega\left(U_{a}\right) \wedge \bar{\Omega}\left(\bar{U}_{a}\right)\right)-\log (-i(\tau-\bar{\tau})), \\
& =-\log \left(i\left(\bar{\omega}_{a} \mathcal{G}_{a}-\omega_{a} \overline{\mathcal{G}}_{a}\right)\right)-\log (-i(\tau-\bar{\tau})), \\
& =-\log \left(-i \Pi^{\dagger} \cdot \Sigma \cdot \Pi\right)-\log (-i(\tau-\bar{\tau})),
\end{aligned}
$$

\footnotetext{
${ }^{1}$ For explicitly constructed vacua on two different two parameter models in the vicinity of the LandauGinzburg respectively conifold point see [31]. For a study of flux vacua of $X_{3}$ in the context of accidental inflation [32] see [33].

${ }^{2}$ For recent reviews see [40-42].
} 
where in the third line of eq. (2.3) we have introduced the symplectic matrix

$$
\Sigma=\left(\begin{array}{cc}
0 & \mathbb{1} \\
-\mathbb{1} & 0
\end{array}\right)
$$

and used the intersection formula

$$
\int_{X_{3}} X \wedge Y=\sum_{a=0}^{h^{2,1}}\left(\int_{A_{a}} X \int_{B^{a}} Y-\int_{A_{a}} Y \int_{B^{a}} X\right),
$$

for general three-forms $X$ and $Y$.

The Gukov-Vafa-Witten flux superpotential is determined by the RR and NS flux $F_{3}$ and $H_{3}$ via [43]

$$
W_{0}=\frac{1}{2 \pi} \int_{X_{3}}\left(F_{3}-\tau H_{3}\right) \wedge \Omega\left(U_{a}\right) .
$$

Due to the quantization of the three-form flux

$$
\begin{aligned}
& \frac{1}{(2 \pi)^{2} \alpha^{\prime}} \int_{A_{a}} F_{3}=f_{1_{a}} \in \mathbb{Z}, \quad \frac{1}{(2 \pi)^{2} \alpha^{\prime}} \int_{B^{a}} F_{3}=f_{2_{a}} \in \mathbb{Z}, \\
& \frac{1}{(2 \pi)^{2} \alpha^{\prime}} \int_{A_{a}} H_{3}=h_{1 a} \in \mathbb{Z}, \quad \frac{1}{(2 \pi)^{2} \alpha^{\prime}} \int_{B^{a}} H_{3}=h_{2 a} \in \mathbb{Z},
\end{aligned}
$$

eq. (2.6) can be written as

$$
W_{0}=2 \pi\left[\left(f_{1 a}-\tau h_{1 a}\right) \mathcal{G}_{a}-\left(f_{2 a}-\tau h_{2 a}\right) U_{a}\right],
$$

where we have set $\alpha^{\prime}=1$ and used again eq. (2.5) and the definition of the periods eq. (2.2).

The D3-tadpole induced by turning on RR and NS flux is given by

$$
L=\frac{1}{(2 \pi)^{4}\left(\alpha^{\prime}\right)^{2}} \int_{X_{3}} H_{3} \wedge F_{3}=h \cdot \Sigma \cdot f=h_{1} f_{2}-h_{2} f_{1} .
$$

The $\mathcal{N}=1$ supergravity scalar potential is given as

$$
V=e^{K}\left(K^{\alpha \bar{\beta}} D_{\alpha} W \overline{D_{\beta} W}-3|W|^{2}\right)
$$

where $K=K_{\mathrm{cs}}+K_{\mathrm{k}}$ and

$$
K_{\mathrm{k}}=-2 \log \mathcal{V}
$$

is the Kähler potential of the Kähler moduli up to corrections in $\alpha^{\prime}$ and $g_{s}$ with $\mathcal{V}$ the volume of the Calabi-Yau $X_{3}$. The indices $\alpha$ and $\beta$ in eq. (2.10) run over the dilaton, the $h^{2,1}$ complex structure moduli and the $h^{1,1}$ Kähler moduli. At tree-level, eq. (2.10) obeys a no-scale structure $[8,9]$ in the Kähler sector:

$$
K^{i \bar{\jmath}} D_{i} W \overline{D_{j} W}=3|W|^{2}, \quad \text { for } i, j=1, \ldots, h^{1,1},
$$

such that

$$
V=e^{K} K^{c \bar{d}} D_{c} W \overline{D_{d} W}
$$


where the indices $c$ and $d$ run over the moduli $\tau$ and $U_{a}$. The no-scale structure eq. (2.12) is broken by $\alpha^{\prime}$ corrections [10] and string loop corrections [11] in $K$, as well as nonperturbative corrections to the superpotential and $K$. However, these corrections are parametrically small in every moduli stabilization scenario where the overall volume of $X_{3}$ is large. Hence, the scalar potential for the dilaton and complex structure moduli eq. (2.13) is positive semi-definite in this limit and a supersymmetric extremum given by a solution to the system of equations

$$
D_{\tau} W=0 \quad \text { and } \quad D_{U_{a}} W=0, \quad \text { for } a=1, \ldots, h^{2,1},
$$

will always be a minimum, i.e. all eigenvalues of the second derivative matrix $V_{a b}$ are positive [13].

Note that due to the appearance of the symplectic matrix, the tadpole eq. (2.9) is at first not positive definite. However, as has been discussed in [5, 24], imposing the supersymmetry conditions $D_{a} W=0$ results in $G_{3}=F_{3}-\tau H_{3}$ being imaginary self-dual (ISD). Since the ISD component of $G_{3}$ always results in positive semi-definite contributions to the tadpole while the anti-ISD component of $G_{3}$ always yields negative semi-definite contributions, a supersymmetric point always has $L \geq 0$. This can be seen nicely if the ISD condition is displayed as [44]

$$
*_{6} s H_{3}=-\left(F_{3}-\sigma H_{3}\right),
$$

i.e. only $h^{2,1}+1$ of the original $2 h^{2,1}+2$ fluxes are independent once the ISD condition is invoked and

$$
L \sim \int_{X_{3}} H_{3} \wedge F_{3} \sim \int_{X_{3}} H_{3} \wedge *_{6} H_{3} \sim \int_{X_{3}} \sqrt{\tilde{g}}\left|H_{3}\right|^{2}>0,
$$

where we have used eq. (2.15).

The type IIB ten dimensional effective supergravity Lagrangian is invariant under $\mathrm{SL}(2, \mathbb{Z})$ transformations

$$
\begin{aligned}
\tau & \rightarrow \frac{a \tau+b}{c \tau+d} \quad \text { with } \quad a, b, c, d \in \mathbb{Z} \quad \text { and } \quad a d-b c=1, \\
\left(\begin{array}{c}
H_{3} \\
F_{3}
\end{array}\right) & \rightarrow\left(\begin{array}{ll}
d & c \\
b & a
\end{array}\right) \cdot\left(\begin{array}{c}
H_{3} \\
F_{3}
\end{array}\right)
\end{aligned}
$$

which implies

$$
G_{3} \rightarrow \frac{G_{3}}{c \tau+d} .
$$

As is easily verified, these transformations also leave the D3 tadpole eq. (2.9) invariant. When determining the solution space of flux vacua of $X_{3}$ we have to make sure to consider only inequivalent vacua under the transformations eq. (2.17).

\subsection{Effective reduction of the moduli space}

Consider the two parameter $\psi, \phi$-family of threefolds $\mathbb{C P}_{11169}^{4}[18]$ given by the vanishing of the polynomials

$$
x_{1}^{18}+x_{2}^{18}+x_{3}^{18}+x_{4}^{3}+x_{5}^{2}-18 \psi x_{1} x_{2} x_{3} x_{4} x_{5}-3 \phi x_{1}^{6} x_{2}^{6} x_{3}^{6},
$$


i.e. all except two of the 272 complex structure moduli which correspond to monomials in the general degree 18 Calabi-Yau hypersurface equation have been set to zero. As was discussed in [25], eq. (2.19) is invariant under a global $\Gamma=\mathbb{Z}_{6} \times \mathbb{Z}_{18}$ symmetry. This symmetry is used in the Greene-Plesser construction [26] to construct the mirror Calabi-Yau which in this case has $h^{1,1}=272$ and $h^{2,1}=2$. Furthermore, the moduli $\psi$ and $\phi$ in eq. (2.19) describe the two complex structure moduli of this mirror manifold. As was pointed out in [45], the periods of the mirror agree with those of $\mathbb{C P}_{11169}^{4}[18]$ at the $\Gamma$ symmetric point. Also, [45] shows that the complete set of $h^{2,1}$ complex structure moduli can be divided into a $\Gamma$-invariant subspace and its complement. The moduli with trivial transformation are exactly those that do not vanish at the $\Gamma$ symmetric point, in this case $\psi$ and $\phi$.

To make use of the agreement of the prepotential for the complex structure sector of $\mathbb{C P}_{1169}^{4}[18]$ and its mirror in the large complex structure limit, it is useful to introduce the complex coordinates $U_{1}$ and $U_{2}$ that are related to $\psi$ and $\phi$ as [25]

$$
\begin{aligned}
X_{1}=-\frac{1}{q_{1}}( & 1+312 q_{1}+2 q_{2}+10260 q_{1}^{2}-540 q_{1} q_{2}-q_{2}^{2} \\
& \left.-901120 q_{1}^{3}+120420 q_{1}^{2} q_{2}+20 q_{2}^{3}+\ldots\right) \\
X_{2}=-\frac{1}{q_{2}}( & 1+180 q_{1}-6 q_{2}+11610 q_{1}^{2}+180 q_{1} q_{2}+27 q_{2}^{2} \\
& \left.+514680 q_{1}^{3}-150120 q_{1}^{2} q_{2}-5040 q_{1} q_{2}^{2}-164 q_{2}^{3}+\ldots\right),
\end{aligned}
$$

up to third order in the $q_{j} \equiv e^{2 \pi i U_{j}}$ with the large complex structure coordinates

$$
X_{1}=\frac{(18 \psi)^{6}}{3 \phi} \quad \text { and } \quad X_{2}=(3 \phi)^{3} \text {. }
$$

The large complex structure limit corresponds to $X_{j} \rightarrow \infty$ which is equivalent to $\operatorname{Im}\left(U_{j}\right) \rightarrow$ $\infty$ as can be seen from eq. (2.20).

There are two conifold singularities given by the equations [25]

$$
\begin{array}{ll}
\mathrm{CF}_{1}:\left(26244 \psi^{6}+\phi\right)^{3}=1 & \Leftrightarrow \frac{X_{2}}{27}\left(\frac{X_{1}}{432}+1\right)^{3}=1, \\
\mathrm{CF}_{2}: \phi^{3}=1 & \Leftrightarrow \frac{X_{2}}{27}=1 .
\end{array}
$$

Let us come back to the problem of finding supersymmetric extrema by solving eq. (2.14). As was noted in $[28,46]$, to find an extremum it is sufficient to turn on fluxes only along the six $\Gamma$-invariant three-cycles, i.e.

$$
f=\left(f_{1_{1}}, f_{1_{2}}, f_{1_{3}}, f_{2_{1}}, f_{2_{2}}, f_{2_{3}}, 0, \ldots, 0\right) \quad \text { and } \quad h=\left(h_{1_{1}}, h_{1_{2}}, h_{1_{3}}, h_{2_{1}}, h_{2_{2}}, h_{2_{3}}, 0, \ldots, 0\right),
$$

having set to zero all the components along the $b_{3}-6$ non-invariant three-cycles. It is then possible to achieve $D_{a} W=0$ for all 272 complex structure moduli, ${ }^{3}$ and hence to

\footnotetext{
${ }^{3}$ Note that orientifolding will project out some of the 272 complex structure moduli. Since the exact number of projected out directions depends on the position of the O-plane we stick to the upper bound of 272 for a general treatment.
} 
find a minimum of the positive definite tree-level no-scale scalar potential eq. (2.13). This is due to the fact that, for this $\Gamma$ invariant flux, the symmetry $\Gamma$ is realized at the level of the four-dimensional effective action. Note that the restriction to flux on the $\Gamma$ invariant cycles is purely for simplicity, as the analysis of the complete 272 dimensional complex structure moduli space is practically extremely challenging.

Let us explain in more detail why the flux vector in eq. (2.23) generically provides a stable minimum of all 272 complex structure moduli $[28,46]$. We first consider $D_{\tilde{U}_{a}} W_{0}=0$, where $\tilde{U}_{a}$ for $a=3, \ldots, 272$ denote the non-trivially transforming moduli under $\Gamma=\mathbb{Z}_{6} \times \mathbb{Z}_{18}$. In the large complex structure limit, the prepotential $\mathcal{G}$ is a polynomial function of all $h^{2,1}$ complex structure moduli that has to transform trivially under $\Gamma$, since if it would not, $\Gamma$ could be used to fix the non-trivially transforming moduli. ${ }^{4}$ Hence, the non-trivially transforming $\tilde{U}_{a}$ have to appear at least quadratic in $\mathcal{G}$ in order to represent a $\Gamma$ invariant contribution to $\mathcal{G}$. This information, together with having switched on flux only along the $\Gamma$ invariant directions, is sufficient to show

$$
W_{0, \tilde{U}_{a}}=K_{\tilde{U}_{a}}=0 \quad \text { at } \quad \tilde{U}_{a}=0 \quad \text { for } \quad a=3, \ldots, 272,
$$

since $W_{0, \tilde{U}_{a}}$ is a polynomial function which is at least linear in the $\tilde{U}_{a}$, see eq. (2.8) and $K_{\tilde{U}_{a}}$ is a rational function which is at least linear in the numerator in the $\tilde{U}_{a}$, see eq. (2.3). Hence, $D_{\tilde{U}_{a}} W_{0}=W_{0, \tilde{U}_{a}}+K_{\tilde{U}_{a}} W_{0}=0$ at $\tilde{U}_{a}=0$ for $a=3, \ldots, 272$. This reduces the full set of conditions $D_{a} W=0 \forall a$ to the three equations

$$
\left.D_{I} W\right|_{\tilde{U}_{a}=0}=0 \quad \text { for } I=\tau, U_{1}, U_{2} .
$$

This is equivalent to set $\tilde{U}_{a}=0$ from the beginning and study the stabilization problem for the reduced case with two complex structure moduli, as we do in the following.

In [25], the prepotential $\mathcal{G}$ for the two complex structure moduli $U_{1}=\omega_{1} / \omega_{0}$ and $U_{2}=\omega_{2} / \omega_{0}$ was derived via mirror symmetry in the large complex structure limit to be

$$
\mathcal{G}\left(\omega_{0}, \omega_{1}, \omega_{2}\right)=\xi \omega_{0}^{2}+\frac{17 \omega_{0} \omega_{1}}{4}+\frac{3 \omega_{0} \omega_{2}}{2}+\frac{9 \omega_{1}^{2}}{4}+\frac{3 \omega_{1} \omega_{2}}{2}-\frac{9 \omega_{1}^{3}+9 \omega_{1}^{2} \omega_{2}+3 \omega_{1} \omega_{2}^{2}}{6 \omega_{0}},
$$

with $\xi=\frac{\zeta(3) \chi}{2(2 \pi i)^{3}} \simeq-1.30843 i$ determined by the Euler number $\chi$ of the Calabi-Yau.

Eq. (2.26) receives instanton corrections which are given as

$$
\begin{aligned}
\mathcal{G}_{\text {inst. }}\left(q_{1}, q_{2}\right)=\frac{1}{(2 \pi i)^{3}} & \left(540 q_{1}+\frac{1215 q_{1}^{2}}{2}+560 q_{1}^{3}+3 q_{2}-1080 q_{1} q_{2}+143370 q_{1}^{2} q_{2}\right. \\
& \left.-\frac{45 q_{2}^{2}}{2}+2700 q_{1} q_{2}^{2}+\frac{244 q_{2}^{3}}{9}+\ldots\right)
\end{aligned}
$$

with $q_{a}=\exp \left(2 \pi i U_{a}\right)$ and we have set $\omega_{0}=1$. The dots in eq. (2.27) denote higher powers in the $q_{a}$ which are suppressed in the large complex structure limit $u_{a}=\operatorname{Im}\left(U_{a}\right) \gtrsim 1$. We define the large complex structure limit via

$$
\frac{\left|\mathcal{G}_{\text {inst. }}\right|}{|\mathcal{G}|} \leq \epsilon_{\mathrm{LCS}}, \quad \frac{540 e^{-2 \pi u_{1}}}{(2 \pi)^{3}|\mathcal{G}|} \leq \epsilon_{\mathrm{LCS}} \quad \text { and } \quad \frac{3 e^{-2 \pi u_{2}}}{(2 \pi)^{3}|\mathcal{G}|} \leq \epsilon_{\mathrm{LCS}}
$$

\footnotetext{
${ }^{4} \mathcal{G}$ completely determines the moduli space of the (before orientifolding) $\mathcal{N}=2$ moduli space. If it would not be invariant the complex structure moduli space would have been reduced, i.e. some flat directions lifted but this does not happen just because there exists a $\Gamma$ symmetric point.
} 
for small $\epsilon_{\mathrm{LCS}}$. The two last conditions in eq. (2.28) are imposed to ensure that there are no cancellations between the terms in $\mathcal{G}_{\text {inst. }}$, i.e. the leading correction in $e^{-2 \pi u_{a}}$ is actually small. Furthermore, to have a valid description of the complex structure moduli by $\mathcal{G}$ we have to ensure that we are not in the vicinity of the conifold points eq. (2.22), i.e.

$$
\left|\frac{X_{2}}{27}\left(\frac{X_{1}}{432}+1\right)^{3}-1\right| \geq \epsilon_{C F} \quad \text { and } \quad\left|\frac{X_{2}}{27}-1\right| \geq \epsilon_{C F}
$$

with small $\epsilon_{C F}$.

\section{The polynomial homotopy continuation method}

We want to solve the non-linear eqs. (2.25) derived from the prepotential eq. (2.26) for the 6 real variables $x_{i}=u_{1}, u_{2}, s, \nu_{1}, \nu_{2}$ and $\sigma$. The parameters of these equations are the 12 fluxes $f_{1}, f_{2}, h_{1}$ and $h_{2}$ in eq. (2.23). Though systems of non-linear equations are extremely difficult to solve in general, if the non-linearity in the system is polynomial-like, then the recently developed algebraic geometry methods can rescue the situation. In particular, we use the so-called numerical polynomial homotopy continuation (NPHC) method [29] which finds all the solutions of the given system of polynomial equations. This method has been used in various problems in particle theory and statistical mechanics in refs. [47-57].

\subsection{The algorithm}

Here we briefly explain the NPHC method: for a system of polynomial equations, $P(x)=0$, where $P(x)=\left(p_{1}(x), \ldots, p_{m}(x)\right)^{T}$ and $x=\left(x_{1}, \ldots, x_{m}\right)^{T}$, which is known to have isolated solutions, the Classical Bézout Theorem asserts that for generic values of coefficients, the maximum number of solutions in $\mathbb{C}^{m}$ is $\prod_{i=1}^{m} d_{i}$. Here, $d_{i}$ is the degree of the $i$ th polynomial. This bound, the classical Bézout bound (CBB), is exact for generic values (see $[29,58]$ for details).

Based on the $\mathrm{CBB}$, a homotopy can be constructed as

$$
H(x, t)=\gamma(1-t) Q(x)+t P(x),
$$

where $\gamma$ is a generic complex number and $t \in[0,1) . Q(x)=\left(q_{1}(x), \ldots, q_{m}(x)\right)^{T}$ is a system of polynomial equations with the following properties:

1. the solutions of $Q(x)=H(x, 0)=0$ are known or can be easily obtained. $Q(x)$ is called the start system and the solutions are called the start solutions,

2. the number of solutions of $Q(x)=H(x, 0)=0$ is equal to the CBB for $P(x)=0$,

3. the solution set of $H(x, t)=0$ for $0 \leq t \leq 1$ consists of a finite number of smooth paths, called homotopy paths, each parameterized by $t \in[0,1)$, and

4. every isolated solution of $H(x, 1)=P(x)=0$ can be reached by some path originating at a solution of $H(x, 0)=Q(x)=0$. 
The start system $Q(x)=0$ can for example be taken to be

$$
Q(x)=\left(\begin{array}{c}
x_{1}^{d_{1}}-1 \\
\vdots \\
x_{m}^{d_{m}}-1
\end{array}\right)=0,
$$

where $d_{i}$ is the degree of the $i^{t h}$ polynomial of the original system $P(x)=0$. Eq. (3.2) is easy to solve and guarantees that the total number of start solutions is $\prod_{i=1}^{m} d_{i}$, all of which are non-singular.

We can then track all the paths corresponding to each solution of $Q(x)=0$ from $t=0$ to $t=1$. The paths which reach $P(x)=0=H(x, 1)$ are the solutions of $P(x)=0$. By implementing an efficient path tracker algorithm, all isolated solutions of a system of multivariate polynomials system can be obtained because it is shown [29] that for a generic $\gamma$, there are no singularities (i.e. paths do not cross each other) for $t \in[0,1)$. In this respect, the NPHC method has a great advantage over all other known methods for finding stationary points.

There are several sophisticated numerical packages well-equipped with path trackers such as Bertini [59], PHCpack [60], PHoM [61] and HOM4PS2 [58, 62]. We mainly use Bertini to get the results in this paper.

We mean by a solution a set of values of variables which satisfies the eqs. (2.25) with tolerance $10^{-10}$. All the solutions come with real and imaginary parts. A solution is a real solution if the imaginary part of each of the variables is less than or equal to the tolerance $10^{-6}$ (below which the number of real solutions does not change, i.e. it is robust for the problem at hand). All these solutions can be further refined to an arbitrary precision.

The advantages of the homotopy based on the $\mathrm{CBB}$ are (1) the CBB is easy to compute, and (2) the start system based on the CBB can be solved quickly. The drawback of it is that the CBB does not take the sparsity of the system into account: systems arising in practice have far fewer solutions than the CBB, so a large portion of the computational effort is wasted.

Hence, one can also use homotopies based on tighter upper bounds. For example, one can compute the so-called 2-Homogeneous Bézout Bound or the Bernstein-KhovanskiiKushnirenko bound [63-65] which are tighter upper bounds. These two bounds were explained in ref. [47, 54]. We note that, as with the CBB, the 2 HomBB and BKK bound are also generically sharp with respect to the family of polynomial systems under consideration.

There is yet another, rather more practical, way of solving a parametric system which is called Cheater's homotopy [66, 67]: let us say we want to solve a parametric system, $\vec{f}(\vec{q} ; \vec{x})=\overrightarrow{0}$ where $\vec{x}$ are variables and $\vec{q}$ are parameters, in our case the fluxes. Now, it can be shown $[66,67]$ that the maximum number of complex solutions at any parameter point is the number of solutions at a generic parametric point. So our strategy is first to solve the system at a generic parameter point and then using the solutions at this point as the start solutions for the systems at all other parameter-points. This homotopy is called cheater's homotopy. A recently developed software, based on Bertini, called Paramotopy [68], precisely uses cheater's homotopy and goes over a huge number of parameter points in parallel. The 
package is publicly not available yet though the respective research group has kindly given access to the code for the purpose of the computation in this paper. We will publish the details on the cheater's homotopy and the package Paramotopy elsewhere [69].

\subsection{The scan}

We define a set of flux parameters on which we apply the algorithm described in the previous section 3.1. Since we are only interested in supersymmetric flux vacua, we can make use of the ISD condition eq. (2.15) and define a flux configuration via

$$
H_{3}=\left(\begin{array}{c}
h_{1} \\
h_{2}
\end{array}\right) \quad \text { and } \quad F_{3}=\left(\begin{array}{c}
-h_{2} \\
h_{1}
\end{array}\right),
$$

with $h_{1}, h_{2} \in \mathbb{Z}^{3}$. Note that since we have two complex structure moduli we have initially $2 \cdot 2+2=6$ flux parameters for both $H_{3}$ and $F_{3}$ but the ISD condition eq. (2.15) reduces this to the six parameters given in eq. (3.3). Furthermore, the D3 tadpole eq. (2.9) becomes manifestly positive semi-definite, i.e.

$$
L=h_{1}^{2}+h_{2}^{2} .
$$

To scan efficiently, we apply the paramotopy algorithm only to $\mathrm{SL}(2, \mathbb{Z})$ inequivalent flux configurations. Note that a configuration of the form eq. (3.3) transforms as

$$
H_{3}^{\prime}=\left(\begin{array}{l}
h_{1}^{\prime} \\
h_{2}^{\prime}
\end{array}\right)=\left(\begin{array}{l}
d h_{1}-c h_{2} \\
d h_{2}+c h_{1}
\end{array}\right) \quad \text { and } \quad F_{3}^{\prime}=\left(\begin{array}{l}
f_{1}^{\prime} \\
f_{2}^{\prime}
\end{array}\right)=\left(\begin{array}{c}
b h_{1}-a h_{2} \\
b h_{2}+a h_{1}
\end{array}\right),
$$

under $\mathrm{SL}(2, \mathbb{Z})$ transformations, eq. (2.17). For general $a, b, c$ and $d, F_{3}^{\prime}$ in eq. (3.5) will not be of the form in eq. (3.3) but only for the 4 cases

$$
a= \pm 1, b=0, c=0, d= \pm 1 \quad \text { and } \quad a=0, b= \pm 1, c=\mp 1, d=0 .
$$

This corresponds to the $\mathrm{SL}(2, \mathbb{Z})$ equivalent flux configurations

$$
\left(\begin{array}{l}
h_{1} \\
h_{2}
\end{array}\right) \cong\left(\begin{array}{c}
-h_{1} \\
-h_{2}
\end{array}\right) \cong\left(\begin{array}{c}
-h_{2} \\
h_{1}
\end{array}\right) \cong\left(\begin{array}{c}
h_{2} \\
-h_{1}
\end{array}\right) .
$$

Note that the two transformations on the l.h.s. of eq. (3.6) do not transform the dilaton $\tau^{\prime}=\tau$ while the two transformations on the r.h.s. act as $\tau^{\prime}=-1 / \tau$.

The number of $\mathrm{SL}(2, \mathbb{Z})$ inequivalent flux configurations in a spherical region defined by a spherical constraint eq. (3.4) can be estimated as $\pi^{3} /(4 \Gamma[4])(\sqrt{L})^{6}$, using the formula for the volume of the n-sphere $V_{n}(r)=\pi^{n / 2} / \Gamma(n / 2+1) r^{n}$. The factor $1 / 4$ accounts for the 4 equivalent configurations in eq. (3.7). If we had switched on more flux $n>6$ the number of lattice points grows very rapidly $\sim L^{n / 2}$.

For our scan, we choose $L=34$ such that we scan over 52,329 parameter points (the above estimate yields 55,391). On the FermiLab cluster using 100 nodes each with 32 cores (each core with $2.0 \mathrm{GHz}$ cloak speed), the calculation time in total is around 75, 000 hours, with $60-100$ minutes per parameter point. 


\subsection{Distribution of parameters}

In this section, we want to discuss the distribution of the following parameters as results of the scan defined in the previous section 3.2:

- $u_{1}$ and $u_{2}$, to see how many points reach the large complex structure limit as defined in eq. (2.28).

- $\tau$, to identify regions of weak respectively strong coupling.

- The number of solutions for a given D3 tadpole $L$.

- $W_{0}$, the flux superpotential.

- The masses of the moduli $m^{2}$ and the gravitino mass $m_{3 / 2}^{2}$.

- The available fine-tuning $\Delta \Lambda$ of the cosmological constant $\Lambda$.

- The amount of flux vacua for which a dS vacuum can be constructed via Kähler uplifting in 3.4.

For the 52,329 parameter points, we find a total of 531,370 solutions to the eqs. (2.25). This corresponds to an average of 10.2 solutions per parameter point. For 1,360 parameter points we do not find a solution. Many of the solutions are unphysical and hence have to be sorted out: a subset of 288,160 fulfill the criterion of a physical string coupling $g_{s}>0$ and only a subset of 26,297 respective 16,235 is in accordance with the large complex structure criterion eq. (2.28) for $\epsilon_{\mathrm{LCS}}=10^{-1}$ respective $\epsilon_{\mathrm{LCS}}=10^{-2}$. Of these none have to be sorted out because they are in the vicinity of the conifold singularities eq. (2.29) having chosen $\epsilon_{C F}=10^{-2}$. Due to the strong suppression of the large complex structure limit in the general solution space of eq. (2.25), the minimal flux method has the advantage of directly searching for solutions in this region.

For the distribution of the dilaton, we can use $\mathrm{SL}(2, \mathbb{Z})$, to transform each solution to the fundamental domain

$$
-\frac{1}{2} \leq \operatorname{Re}(\tau) \leq \frac{1}{2} \quad \text { and } \quad|\tau|>1,
$$

via the successive transformations

$$
\tau^{\prime}=\tau+b, \quad G_{3}^{\prime}=G_{3},
$$

i.e. $a=1, b \in \mathbb{Z}, c=0, d=1$ and

$$
\tau^{\prime}=-1 / \tau, \quad G_{3}^{\prime}=G_{3} / \tau,
$$

i.e. $a=0, b=-1, c=1, d=0$.

We show the distribution of the obtained values for $\tau=\sigma+i s$ in figure 2. We see that the strongly coupled region $s=1 / g_{s} \sim 1$ is preferred and large values of $s>10$ are obtained for a fraction of $5 \%$. 


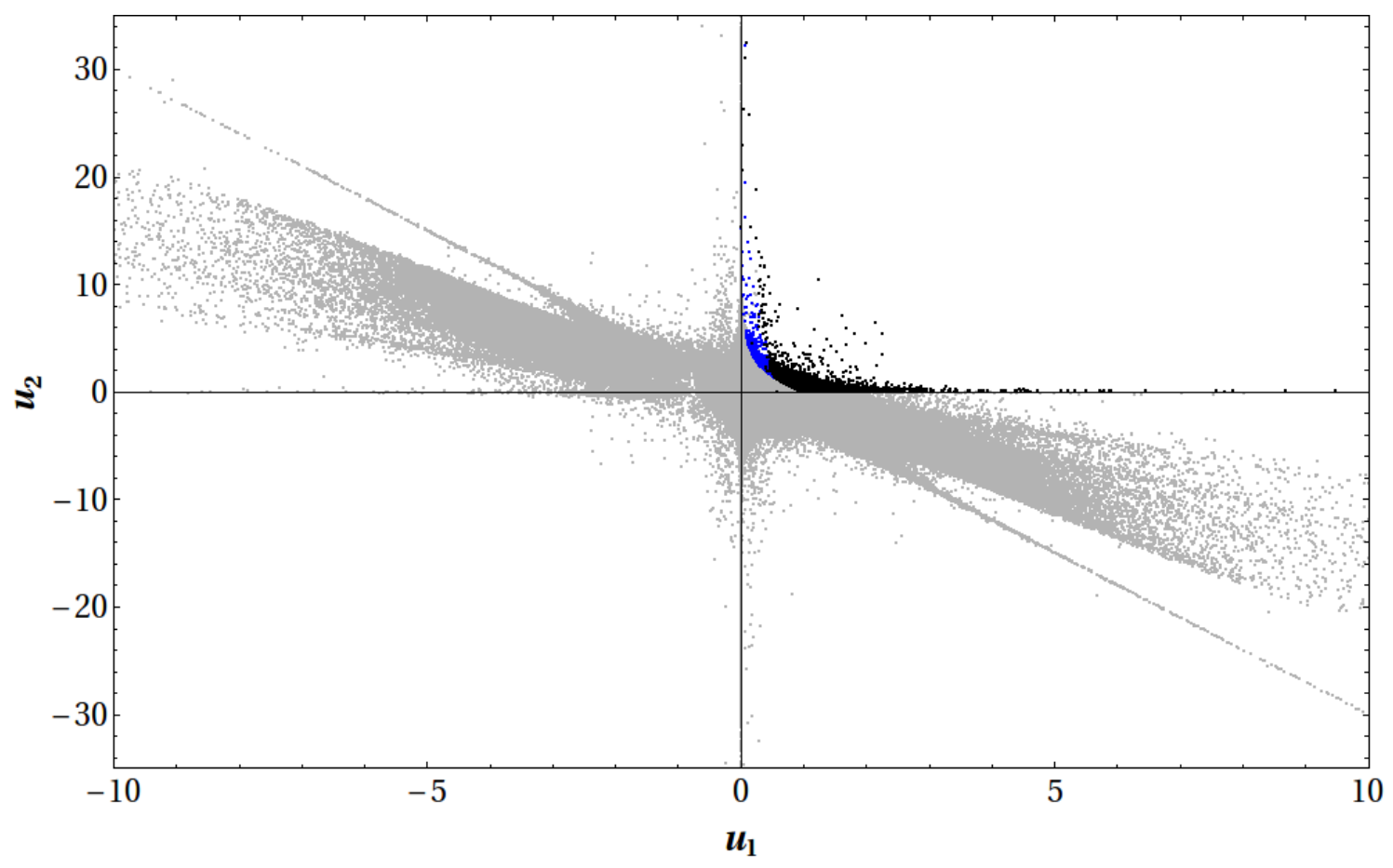

Figure 1. Distribution of $u_{1}$ and $u_{2}$ for the complete set of solutions to eqs. (2.25) (gray) and for the physical solutions fulfilling the criterion for the validity of the large complex structure limit (blue for $\epsilon_{\mathrm{LCS}}=10^{-1}$ and black for $\epsilon_{\mathrm{LCS}}=10^{-2}$ ), eq. (2.28).

The number of vacua of $X_{3}$ in the large complex structure limit for a given D3 tadpole $L$ was estimated in [28] ss $^{5}$

$$
N_{\mathrm{vac}}=\frac{(2 \pi L)^{3}}{3 !} \int \operatorname{det}(-\mathcal{R}-\mathbf{1} \cdot \omega)
$$

with Kähler form $\omega$ and the curvature two-form $\mathcal{R}$ of the moduli space. The integral in eq. (3.11) was estimated in [28] to be $1 / 1296$, using the $\Gamma$ symmetry of the moduli space such that

$$
N_{\mathrm{vac}} \simeq 0.03 L^{3}
$$

Since paramotopy allows us to find all solutions for a given flux configuration we can not only check the $L$ dependence of eq. (3.11) but also the normalization. This depends on the value chosen for $\epsilon_{\mathrm{LCS}}$, i.e. a greater $\epsilon_{\mathrm{LCS}}$ will yield a larger normalization factor. Fitting the number of solutions with $h^{2} \leq L$ in the large complex structure limit to the tadpole $L$ we find

$$
\begin{array}{lll}
N_{\mathrm{vac}} \simeq(0.50 \pm 0.04) L^{2.94 \pm 0.03} & \text { for } & \epsilon_{\mathrm{LCS}}=10^{-2} \\
N_{\mathrm{vac}} \simeq(0.85 \pm 0.06) L^{2.93 \pm 0.03} & \text { for } & \epsilon_{\mathrm{LCS}}=10^{-1}
\end{array}
$$

\footnotetext{
${ }^{5}$ Note that $N_{\mathrm{vac}} \sim L^{6}$ in [28] which is due to the fact that 12 independent fluxes have been switched on while we effectively switch on 6 independent fluxes, see eq. (3.3).
} 


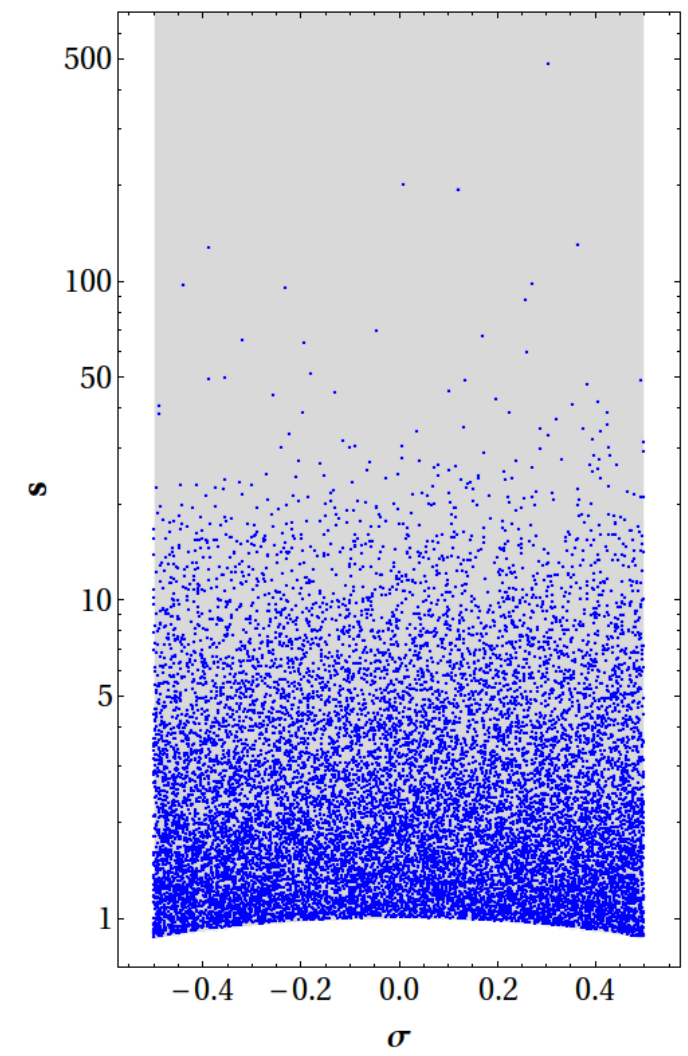

Figure 2. Distribution of $\tau$ for the paramotopy scan with $\epsilon_{\mathrm{LCS}}=10^{-2}$.

The dependence of $N_{\text {vac }}$ on $L$ and the fit in eq. (3.13) are shown in figure 3. Considering the very general arguments that are used to derive the estimate eq. (3.11), the agreement within an order of magnitude with the factual number of vacua strongly confirms the statistical analysis of $[24,30]$. In the following, we set $\epsilon_{\mathrm{LCS}}=10^{-2}$.

The distribution of the flux superpotential is shown in figure 3 . We find that for most vacua $\mathcal{O}\left(10^{1}-10^{3}\right)$ values are preferred. To calculate the masses of the moduli we have to know the value of the volume $\mathcal{V}$ of $X_{3}$ which enters via the Kähler potential of the Kähler moduli given in eq. (2.11). Note that we have not specified the stabilization mechanism for the Kähler moduli and hence have no information about the value of $\mathcal{V}$. For the KKLT and Kähler uplifting scenarios the volume is typically stabilized at $\mathcal{O}\left(10^{2}-10^{4}\right)$ while for the LVS it is $\mathcal{O}\left(10^{6}-10^{15}\right)$. Hence, we can only calculate the physical masses $m$ up to factors of $\mathcal{V}^{-1}$, i.e.

$$
m=\frac{m_{\mathrm{cs}}}{\mathcal{V}}
$$

where $m_{\mathrm{cs}}$ is the mass calculated from the effective theory of the complex structure moduli only, i.e. $K=K_{\mathrm{cs}}$ and $W=W_{0}$.

The distribution of the physical moduli masses $m^{2}$ in terms of $m_{\mathrm{cs}}^{2}$, i.e. the eigenvalues of the Hessian $\partial_{a} \partial_{b} V$ of the no-scale potential eq. (2.13) for $a, b=u_{1}, u_{2}, s, \nu_{1}, \nu_{2}, \sigma$ is shown 

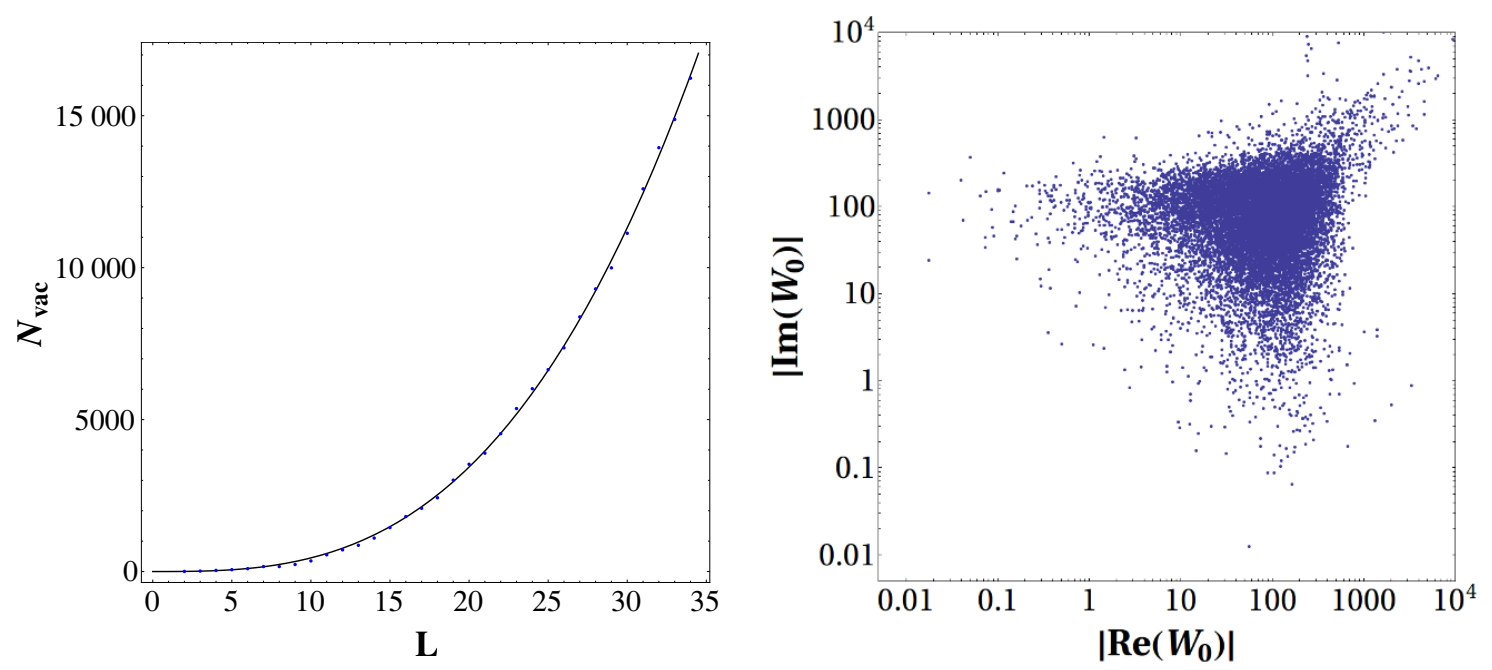

Figure 3. The number of vacua $N_{\text {vac }}$ with $h^{2}<L$ (left) and the logarithmic distribution of the flux superpotential $W_{0}$ (right) in the large complex structure limit with $\epsilon_{\mathrm{LCS}}=10^{-2}$.

in figure 4 as well as the gravitino mass $m_{3 / 2}^{2}$ in terms of the quantity

$$
m_{\mathrm{cs}, 3 / 2}^{2} \equiv m_{3 / 2}^{2} \mathcal{V}^{2}=e^{K_{\mathrm{cs}}}\left|W_{0}\right|^{2} .
$$

This quantity $m_{\mathrm{cs}, 3 / 2}^{2}$ governs the scale of the typical AdS cosmological constant induced by the flux superpotential ignoring the contributions from the Kähler moduli sector.

The distribution of $m_{3 / 2}^{2}$ is peaking at $\left\langle m_{3 / 2}^{2}\right\rangle=3.5 \times 10^{-2} \cdot\left(\frac{100}{\mathcal{V}}\right)^{2}$ with standard deviation $3 \times 10^{-2} \cdot\left(\frac{100}{\mathcal{V}}\right)^{2}$. The complex structure moduli and the dilaton are stabilized at $m^{2} \sim \mathcal{O}\left(10^{-3}-10^{2}\right)\left(\frac{100}{\mathcal{V}}\right)^{2}$. These ranges for the moduli and gravitino masses are compatible with the values obtained for a single explicit flux choice in the same construction $[14,15]$.

The AdS respective dS cosmological constant before tuning is up to $\mathcal{O}(1)$ factors estimated to be

$$
\Lambda \sim \frac{m_{3 / 2}^{2}}{\mathcal{V}}=\frac{m_{\mathrm{cs}, 3 / 2}^{2}}{\mathcal{V}^{3}}
$$

in the LVS respective Kähler uplifting scenarios. In particular, the tunability of $m_{\mathrm{cs}, 3 / 2}$ by three-form flux directly translates into the tunability of the cosmological constant via

$$
\frac{\Delta \Lambda}{\Lambda} \sim 2 \frac{\Delta m_{\mathrm{cs}, 3 / 2}}{m_{\mathrm{cs}, 3 / 2}}
$$

Note that the r.h.s. of eq. (3.18) is independent of the volume $\mathcal{V}$, i.e. fine tuning of $m_{\mathrm{cs}, 3 / 2}$ only has a tiny effect on the VEVs of the Kähler moduli.

Since the polynomial homotopy continuation method allows us to calculate all solutions for a given tadpole $L$ we can estimate $\Delta \Lambda / \Lambda$ by determining the average spacing $\Delta m_{\mathrm{cs}, 3 / 2}$ for all values of $m_{\mathrm{cs}, 3 / 2}$ that are to be found in a $\sigma$-interval around $\left\langle m_{\mathrm{cs}, 3 / 2}\right\rangle$. Since the number of vacua is given as a power-law in $L$, with the exponent linear in the number of flux carrying complex structure moduli $h_{\text {eff }}^{2,1}$, we expect $\Delta m_{\mathrm{cs}, 3 / 2} / m_{\mathrm{cs}, 3 / 2}$ to be of the form

$$
\frac{\Delta m_{\mathrm{cs}, 3 / 2}}{m_{\mathrm{cs}, 3 / 2}} \sim \frac{C}{L^{a\left(h_{\mathrm{eff}}^{2,1}+1\right)}},
$$



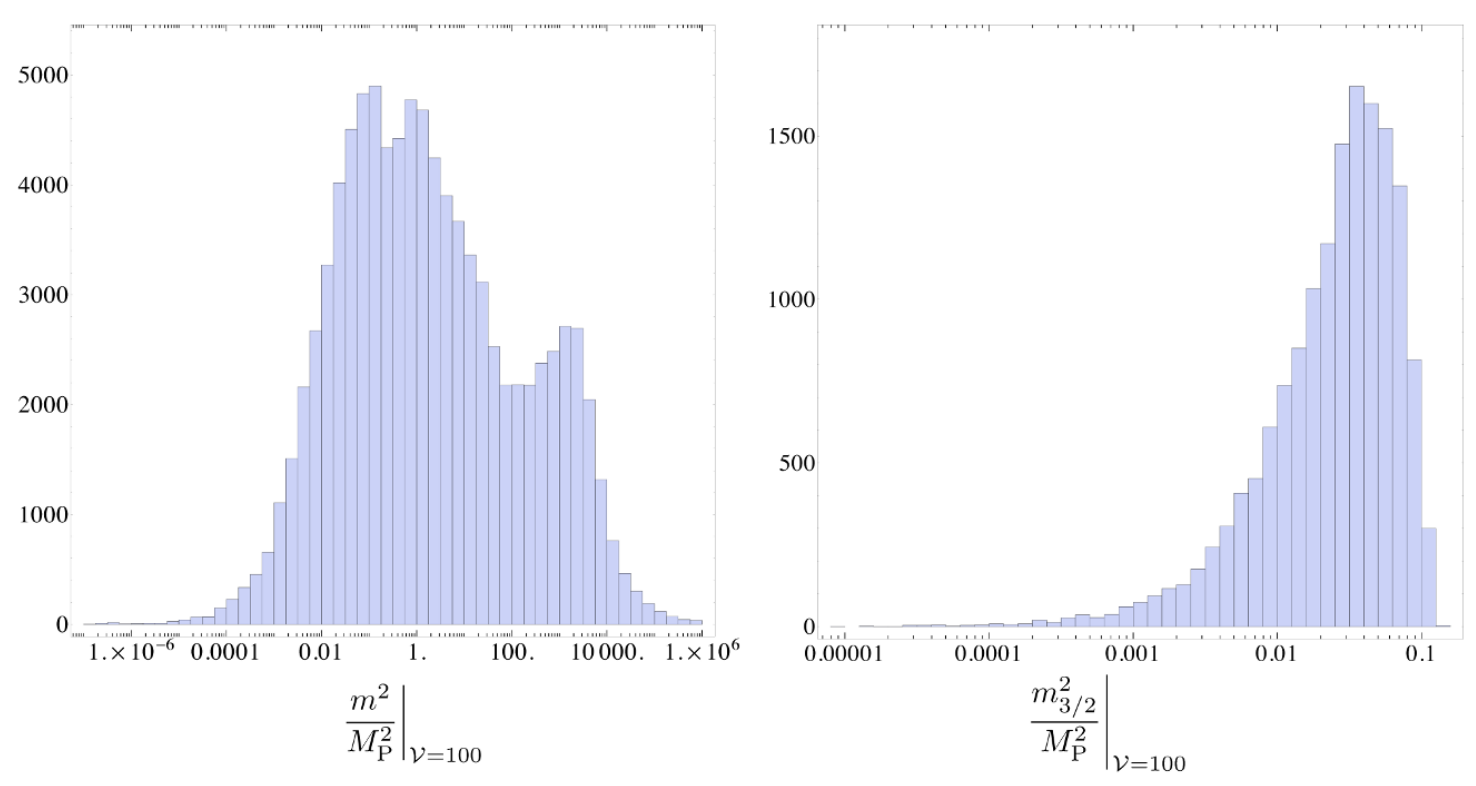

Figure 4. Masses $\left.\frac{m^{2}}{M_{\mathrm{P}}^{2}}\right|_{\mathcal{V}=100}$ of the moduli, and the gravitino mass $\left.\frac{m_{3 / 2}^{2}}{M_{\mathrm{P}}^{2}}\right|_{\mathcal{V}=100}$, for a Calabi-Yau volume $\mathcal{V}=100$. For different Calabi-Yau volumes the masses scale as $\left.\frac{m^{2}}{M_{\mathrm{P}}^{2}}\right|_{\mathcal{V}=100}\left(\frac{100}{\mathcal{V}}\right)^{2}$ for the moduli $u_{1}, u_{2}, s, \nu_{1}, \nu_{2}, \sigma$ (left) and for the gravitino mass as $\left.\frac{m_{3 / 2}^{2}}{M_{\mathrm{P}}^{2}}\right|_{\mathcal{V}=100}\left(\frac{100}{\mathcal{V}}\right)^{2}$ (right). The left plot includes all eigenvalues of the Hessian, i.e. 6 values per parameter point.

with $C, a>0$. We can determine these parameters by fitting the l.h.s. of eq. (3.19) as a function of $L$ for $h_{\text {eff }}^{2,1}=2$. Choosing a $3-\sigma$ interval $^{6}$ around $\left\langle m_{\mathrm{cs}, 3 / 2}\right\rangle$ we find the available tuning for the cosmological constant to be

$$
\frac{\Delta \Lambda}{\Lambda} \simeq(6.0 \pm 0.3) L^{-(0.95 \pm 0.005)\left(h_{\mathrm{eff}}^{2,1}+1\right)},
$$

where we have included the statistical errors of the fit parameters $C$ and $a$.

Let us assume that eq. (3.20) is valid and $\left\langle m_{\mathrm{cs}, 3 / 2}\right\rangle \sim \mathcal{O}(10)$ also for larger values of $L \sim$ $\mathcal{O}\left(10^{3}\right)$ and larger values of $h_{\text {eff }}^{2,1} \sim \mathcal{O}\left(10^{1}-10^{2}\right) .{ }^{7}$ Then, we can extrapolate the values of the cosmological constant eq. (3.17) and its tunability to more realistic scenarios, see table 1.

To tune the cosmological constant to the accuracy given in table 1 , one has to make the assumption that every supersymmetric and hence tachyon-free flux vacuum, remains tachyon-free after stabilizing the Kähler moduli and uplifting which breaks supersymmetry. For generic $\mathcal{N}=1$ supergravity scalar potentials there could be strong suppressions of tachyonic free configurations [34-36] for large values of $h^{1,1}$. On the other hand, the noscale property eq. (2.12) of the models under consideration in this paper yields a positive

\footnotetext{
${ }^{6}$ There is only a weak dependence on the width of the interval. For 5 - $\sigma$ the difference in $\Delta m_{\mathrm{cs}, 3 / 2} / m_{\mathrm{cs}, 3 / 2}$ compared to $3-\sigma$ is less than $1 \%$.

${ }^{7}$ This assumption is reasonable when the prepotential $\mathcal{G}$ is of the same structure as eq. (2.26), i.e. we are considering the large complex structure limit away from e.g. conifold singularities via a mirror construction. It may be interesting to consider such examples with $h_{\text {eff }}^{2,1}=h^{1,1}>2$, e.g. by choosing random pre-factors in a general polynomial prepotential of degree 2 in the $\omega_{i}$.
} 


\begin{tabular}{|c|c|c|}
\hline$h_{\text {eff }}^{2,1}$ & $L$ & $\Delta \Lambda / \Lambda$ \\
\hline 2 & 34 & $7 \cdot 10^{-3} \pm 5 \cdot 10^{-4}$ \\
\hline 2 & 500 & $5 \cdot 10^{-5} \pm 4 \cdot 10^{-6}$ \\
\hline 40 & 34 & $3 \cdot 10^{-58} \pm 2 \cdot 10^{-58}$ \\
\hline 40 & 500 & $10^{-102} \pm 10^{-102}$ \\
\hline
\end{tabular}

Table 1. The tunability $\Delta \Lambda / \Lambda$ of the cosmological constant for different values of $h_{\text {eff }}^{2,1}$ and $L$ with statistical errors propagated from eq. (3.20). The untuned values of the cosmological constant are estimated via eq. (3.17) to be $\mathcal{O}\left(10^{-4}-10^{-22}\right)$ in units of $M_{\mathrm{P}}^{4}$ for $\mathcal{V}$ of $\mathcal{O}\left(10^{2}-10^{8}\right)$. The first row of this table is directly calculated from our dataset while the last three rows are obtained as an extrapolation via eq. (3.20).

semi-definite scalar potential for the dilaton and the complex structure moduli eq. (2.13). This is modified by corrections that break the no-scale property, i.e. most importantly non-perturbative corrections to the superpotential and $g_{s}$ and/or $\alpha^{\prime}$ corrections to the Kähler potential. These corrections are suppressed by an additional power of the volume $\mathcal{V}$ of the compactification relative to the terms in eq. (2.13) [13]. Hence, the eigenvalues of the Hessian matrix with respect to the dilaton and complex structure moduli fields will be positive in the large volume limit such that one only has to make sure that the moduli to be stabilized at a lower scale do not induce any tachyonic directions.

In the following section, we will determine how many de Sitter vacua can be constructed from our dataset on $X_{3}$ via the method of Kähler uplifting.

\subsection{De Sitter vacua via Kähler uplifting}

The two Kähler moduli $T_{1}$ and $T_{2}$ of $X_{3}$ can be stabilized in a dS minimum by Kähler uplifting. A globally consistent D7 brane and gauge flux setup that realizes such a dS vacuum has been presented in [15]. The Kähler potential of $T_{1}$ and $T_{2}$ is given as

$$
K=-2 \log \left[\frac{1}{\sqrt{12}}\left(\left(T_{1}+\bar{T}_{1}\right)+\frac{1}{3}\left(T_{2}+\bar{T}_{2}\right)\right)^{3 / 2}-\frac{1}{18}\left(T_{2}+\bar{T}_{2}\right)^{3 / 2}+\frac{1}{2} \hat{\xi}(\tau, \bar{\tau})\right]
$$

with the leading order $\alpha^{\prime}$ correction [10]

$$
\hat{\xi}(\tau, \bar{\tau})=-\frac{\zeta(3) \chi}{4 \sqrt{2}(2 \pi)^{3}}(-i(\tau-\bar{\tau}))^{3 / 2}
$$

with Euler number $\chi=2(2-272)=-540$. To apply the method of Kähler uplifting we need to balance the leading order $\alpha^{\prime}$ correction to the Kähler potential with non-perturbative contributions to the superpotential. These originate from gaugino condensation of an $\mathrm{SU}(24)$ and $\mathrm{SO}(24)$ pure super Yang Mills from respectively $24 \mathrm{D} 7$ branes wrapping the divisors corresponding to $T_{1}$ and $T_{2}$. The induced superpotential is

$$
W=W_{0}+A_{1} e^{-\frac{2 \pi}{24} T_{1}}+A_{2} e^{-\frac{2 \pi}{22} T_{2}} .
$$


By switching on suitable gauge flux it can be shown [15] that $A_{1}, A_{2} \neq 0$. The induced D3 tadpole by this gauge flux and the geometric contributions from the D7 branes and O7 planes is $L=96$ or $L=104$ [15]. ${ }^{8}$ The one-loop determinants $A_{1}$ and $A_{2}$ depend on the complex structure moduli, the dilaton and also potentially D7 brane moduli. The explicit dependence on these moduli is unknown, however for the purpose of Kähler moduli stabilization the values of $A_{1}$ and $A_{2}$ can be assumed to be constant since complex structure moduli are stabilized at a higher scale. Choosing $A_{1}=A_{2}=1$, it was found numerically in [15], that the pairs of $W_{0}$ and $s$ that are suitable to realize a dS vacuum with small positive tree level vacuum energy ${ }^{9}$ lie on the curve

$$
W_{0}^{\mathrm{dS}}(s)=70.2 s^{-2.35} \quad \text { with } \quad s \geq 4 .
$$

To parametrize our missing knowledge of the values of $A_{1}$ and $A_{2}$ we introduce the parameter $\Delta A$ and the scaling relations

$$
W_{0} \rightarrow W_{0} \cdot \Delta A, \quad A_{1} \rightarrow A_{1} \cdot \Delta A, \quad A_{2} \rightarrow A_{2} \cdot \Delta A,
$$

under which the position of a minimum of the potential eq. (2.10) is invariant since $V \rightarrow$ $V \cdot \Delta A^{2}$. For a given uncertainty in the one-loop determinants $\Delta A^{-1} \leq A_{i} \leq \Delta A$ around $A_{1}=A_{2}=1$ we can then define the criterion

$$
\frac{W_{0}^{\mathrm{dS}}(s)}{\Delta A} \leq\left|W_{0}\right| \leq W_{0}^{\mathrm{dS}}(s) \cdot \Delta A \quad \text { and } \quad s \geq 4,
$$

for a given data point $\left(s,\left|W_{0}\right|\right)$ to allow a dS vacuum via Kähler uplifting.

We show the number of Kähler uplifted dS vacua depending on $\Delta A$ in figure 5 . Due to the suppression of weakly coupled vacua $s \gg 1$ and $\mathcal{O}(1)$ values of the superpotential, the number of vacua that can be uplifted to dS via Kähler uplifting is strongly suppressed. For $\Delta A=10$, only 7 , i.e. a fraction of $\sim 10^{-4}$ of the total number of flux vacua allow such an uplifting.

The available tuning of the cosmological constant via fluxes can be estimated again via eq. (3.18). The Kähler moduli stabilization yields a volume of $\mathcal{V} \simeq 50$ [15] such that the untuned cosmological constant is $\Lambda \sim 6 \cdot 10^{-6}$ and

$$
\frac{\Delta \Lambda}{\Lambda} \simeq 0.38 \pm 0.27
$$

for $\Delta A=10$. We remind the reader, that this is calculated for $L=34$ which is the maximal value we reach in our paramotopy scan and hence less than $L=104$ which is maximally allowed by the gauge flux and D7 brane construction realized for a Kähler uplifted dS vacuum in our explicit $\mathbb{C P}_{11169}^{4}[18]$ example.

To summarize this section, the polynomial homotopy continuation method allows us to find all flux vacua for a given D3 tadpole $L$. The number of these vacua is well

\footnotetext{
${ }^{8}$ The two different values of $L$ originate from two different gauge fluxes that can be switched on in order to cancel the Freed-Witten anomaly of the Whitney-brane that cancels the D7-tadpole induced by the O7-planes. For details, see [15].

${ }^{9}$ In this case, small refers to how small we can tune $\langle V\rangle$ by choosing numerical values for $W_{0}$ and $s$ to a certain decimal place and is not related to the tuning of the cosmological constant.
} 

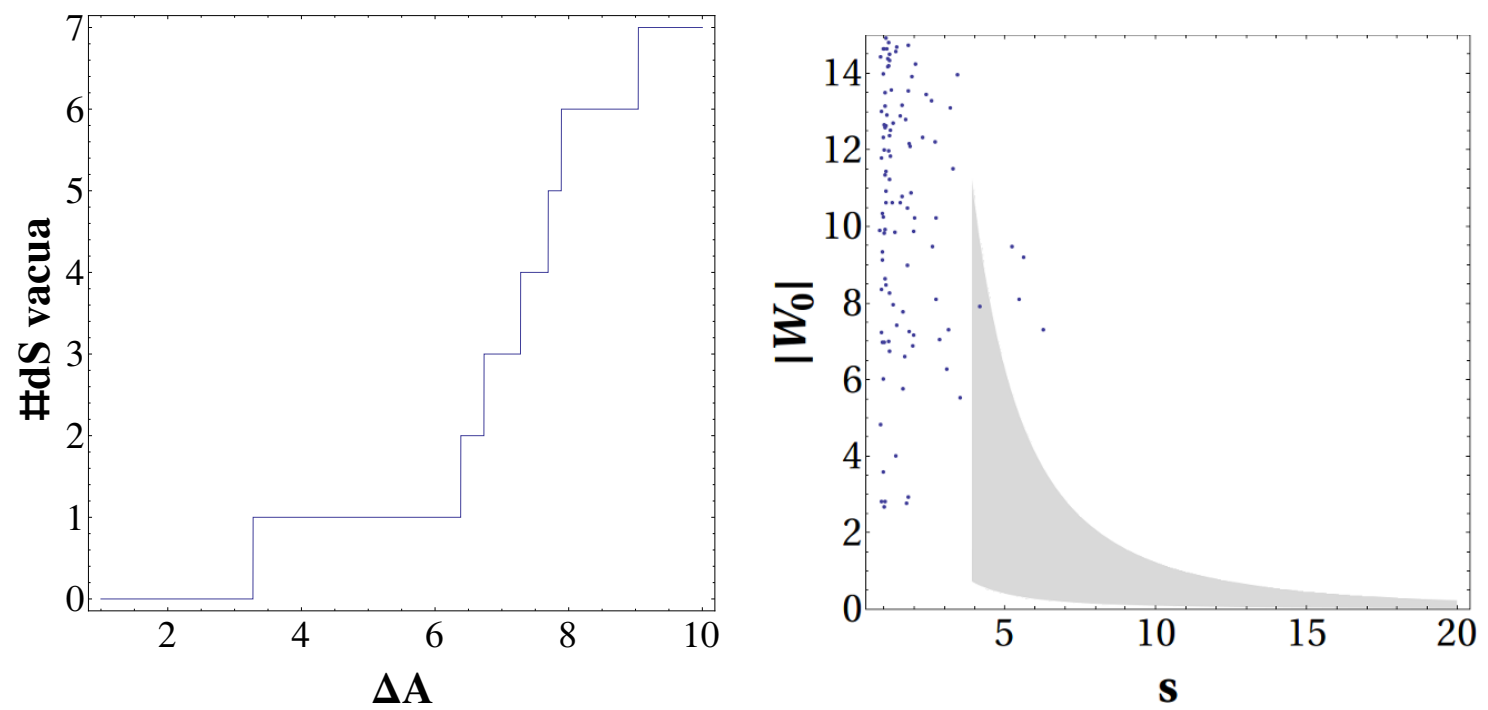

Figure 5. The number of Kähler uplifted dS vacua as a function of $\Delta A$ (left) and data points $\left(s,\left|W_{0}\right|\right)$ (right). Kähler uplifting can be applied in the shaded region $(\Delta A=4)$.

estimated by the statistical analysis of $[24,30]$. We find that strongly coupled vacua $s \gtrsim 1$ are preferred as well as $\mathcal{O}\left(10^{1}-10^{3}\right)$ values of $W_{0}$. Our results can be used to estimate the tunability of the cosmological constant by fluxes and the number of flux vacua that can be Kähler uplifted to a dS vacuum.

\section{The minimal flux method}

In this section we describe the method to find flux vacua that has been first used by Denef, Douglas and Florea [28]. In contrast to the polynomial homotopy continuation method described in section 3, we fix starting values for the VEVs $\left\langle U_{1}\right\rangle_{f i x},\left\langle U_{2}\right\rangle_{f i x}$ and $\langle\tau\rangle_{\text {fix }}$ and solve for the flux values $f$ and $h$.

\subsection{The algorithm}

Due to the linear dependence of $W_{0}$ on $f$ and $h$, see eq. (2.8), the quantities $D_{I} W_{0}=W_{0 I}+K_{I} W_{0}$ for $I=\tau, U_{1}, U_{2}$ are linear in these flux vectors. Hence, if we want to solve the system of equations

$$
\left(W_{0}, D_{\tau} W_{0}, D_{U_{1}} W_{0}, D_{U_{2}} W_{0}\right)=0
$$

this can be written as

$$
M \cdot(f, h)=0,
$$

with $M \in \mathbb{R}^{8 \times 12}$ for general VEVs $\left\langle U_{1}\right\rangle,\left\langle U_{2}\right\rangle,\langle\tau\rangle \in \mathbb{C}$. The dimensions of $M$ are due to the fact that we have 8 real equations in eq. (4.1), and there are 12 flux integers in total in $f$ and $h$. Note that we have also included the condition $W_{0}=0$ in eq. (4.1). In fact, we are not interested in flux vacua where $W_{0}$ is strictly zero since none of the well studied moduli stabilization mechanisms KKLT [6], LVS [13] and Kähler uplifting [12, 14, 15, 70] 
apply in this situation. However, eq. (4.1) will only serve as a starting point and we will eventually end up with vacua where $W_{0} \neq 0$ and $\mathcal{O}(1)$.

For $M \in \mathbb{R}^{8 \times 12}$ there is no hope to find a solution of eq. (4.2) since the flux parameters have to be integers. However, if we neglect instanton corrections induced by eq. (2.27) and choose rational starting values $\left\langle U_{1}\right\rangle,\left\langle U_{2}\right\rangle,\langle\tau\rangle \in \mathbb{Q}+i \mathbb{Q}$ in the superpotential, the only transcendental number in eq. (4.2) is $\xi=\frac{\zeta(3) \chi}{2(2 \pi i)^{3}}=-1.30843 \ldots i$. If we approximate $\xi$ by a rational number $\xi_{\text {rat }}$, for instance $\xi_{\text {rat }}=-13 / 10 i$, we have accomplished $M \in \mathbb{Q}^{8 \times 12}$.

Now, we can hope to find a solution of eq. (4.2) although the entries of $f$ and $h$ will be generically be quite large for generic $M$, since one generally expects them to be at least of the order of the lowest common denominator of the entries of $M$. This puts tension on the D3 tadpole constraint eq. (2.9) since generally the geometry of the compactification manifold and D7 brane configuration generates $L \sim 10^{2}-10^{4}$. We use the same algorithms [71] as the authors of [28], to generate as small as possible values for the entries of $f$ and $h$ in order to generate a not too large D3 tadpole $L<L_{\max }$ where we choose $L_{\max }=500$ to be the maximal value for the D3 tadpole that we consider.

Since the system of equations eq. (4.2) is under determined, the solution space is given by all linear combinations of linearly independent vectors $(f, h)_{i}$ for $i=1, \ldots, 4$, where each $(f, h)_{i}$ is a solution to eq. (4.2), i.e.

$$
(f, h)_{\text {sol }}=\sum_{i=1}^{4} a_{i}(f, h)_{i} \quad \text { with } a_{i} \in \mathbb{Z} .
$$

For obvious practical reasons, we cannot consider all possible values of the $a_{i}$. We find that basis vectors of solutions with $L\left((f, h)_{i}\right)<10$ are extremely rare in our scan. Hence, since $L\left(a_{i}(f, h)_{i}\right)=a_{i}^{2} L\left((f, h)_{i}\right)$, we can safely restrict ourselves to all values of the $a_{i}$ with $-3 \leq a_{i} \leq 3$ in order to fulfill $L\left((f, h)_{\text {sol }}\right)<L_{\max }$.

In the next step, we are looking for solutions to eq. (2.25), including instanton corrections eq. (2.27) to the prepotential and also setting $\xi$ to its transcendental value. We insert the flux solution $(f, h)_{\text {sol }}$ into the equations

$$
\left(D_{\tau} W_{0}, D_{U_{1}} W_{0}, D_{U_{2}} W_{0}\right)=0 \quad \text { (with instanton corrections), }
$$

leaving the VEVs $\left\langle U_{1}\right\rangle,\left\langle U_{2}\right\rangle$ and $\langle\tau\rangle$ unfixed. These are six real equations for six real variables and we can numerically search for a solution in the vicinity of $\left\langle U_{1}\right\rangle_{\text {fix }},\left\langle U_{2}\right\rangle_{\text {fix }}$ and $\langle\tau\rangle_{\text {fix }}$. It has to be checked case by case if the complex structure limit is still valid for these perturbed solutions. The shift of the VEVs from their fixed values may also induce a shift in the superpotential, i.e. $W_{0}$ is not zero anymore. However, note that the value that $W_{0}$ will take in the end is not in any way under our control and it has to be checked if one obtains useful values for the purpose of moduli stabilization.

The above outlined algorithm can be iterated by sampling over a set of VEVs $\left\langle U_{1}\right\rangle_{\text {fix }}$, $\left\langle U_{2}\right\rangle_{\text {fix }}$ and $\langle\tau\rangle_{\text {fix }}$ and approximate $\xi$ values $\xi_{\text {rat }}$.

\subsection{The scan}

First of all, we need to define a set of rational starting values $\left(\left\langle U_{1}\right\rangle,\left\langle U_{2}\right\rangle,\langle\tau\rangle, \xi_{\text {rat }}\right)_{\text {fix }}$ over which the algorithm explained in section 4.1 can be iterated. We set the axionic 


\begin{tabular}{|c|c|c|c|c|}
\hline & $x_{\min }$ & $x_{\max }$ & $q$ & $\#$ \\
\hline$\xi_{\text {rat }}$ & $0.9 \xi$ & $1.1 \xi$ & 20 & 34 \\
\hline$u_{1}$ & 1 & 4 & 5 & 31 \\
\hline$u_{2}$ & $1 / 6$ & 4 & 5 & 40 \\
\hline$s$ & 1 & 10 & 10 & 289 \\
\hline
\end{tabular}

Table 2. Starting values for $\xi_{\text {rat }}$ and the imaginary parts of the moduli. The last column counts the number of elements yielding from the choice of $x_{\min }, x_{\max }$ and $q$.

components of the fixed VEV's to zero, i.e.

$$
\operatorname{Re}\left\langle U_{1}\right\rangle_{f i x}=\operatorname{Re}\left\langle U_{2}\right\rangle_{f i x}=\operatorname{Re}\langle\tau\rangle_{f i x}=0 .
$$

Let $x \in\left[x_{\min }, x_{\max }\right]$ represent $\xi_{\text {rat }}$ and the imaginary parts of the moduli. We use a set of rational numbers that lie in the interval $\left[x_{\min }, x_{\max }\right]$, with the parameter $q$ determining how 'dense' the interval is filled with rational numbers. For example, one has

$$
\left\{1, \frac{5}{4}, \frac{4}{3}, \frac{3}{2}, \frac{5}{3}, \frac{7}{4}, 2\right\}
$$

for $x \in[1,2]$ and $q=4$.

The set of starting values given in table 2 is chosen such that the starting values for the complex structure moduli are in the large complex structure limit eq. (2.28) and the string coupling $g_{s}=1 / s$ is always smaller than one. The total number of points in the grid of starting values defined in table 2 has $12,184,240$ points. Using 80 cores with $2.4 \mathrm{GHz}$ of the DESY Theory Cluster this yields a total calculation time of approximately four weeks.

\subsection{Results}

We find 1,698 solutions fulfilling the constraint $L<L_{\max }=500$ which is only $0.01 \%$ of the total number of points of the scan. Hence, most of the time one can not find a flux vector whose entries are small enough to fulfill the D3 tadpole constraint.

As in section 3.3 , we can make use of the $\mathrm{SL}(2, \mathbb{Z})$ transformations eq. (3.9) and eq. (3.10) to transform every solution to the fundamental domain eq. (3.8). Identifying equivalent solutions in this domain, 1,374 elements of the original solution set are not related via $\mathrm{SL}(2, \mathbb{Z})$ symmetry and hence physically inequivalent. The distribution of $\tau$ is shown in figure 6 . Compared to the paramotopy scan we more easily find weakly coupled vacua with $s \gg 1$ which is due to the fact that we have chosen the starting values for $s$ accordingly, see table 2 .

The distribution of $u_{1}$ and $u_{2}$ as well as the distribution of the superpotential are shown in figure 7 . We find that there are no flux vacua in the vicinity of the conifold singularities eq. (2.29) for $\epsilon_{C F}=10^{-2}$. Also, all flux vacua fulfill the constraint of the validity of the large complex structure limit description, eq. (2.28) for $\epsilon_{\mathrm{LCS}}=10^{-2}$ which is again due to the chosen starting values deep in the large complex structure limit, table 2. Since we solve for vanishing $W_{0}$ in the first step of the algorithm eq. (4.1), we obtain a clustering 


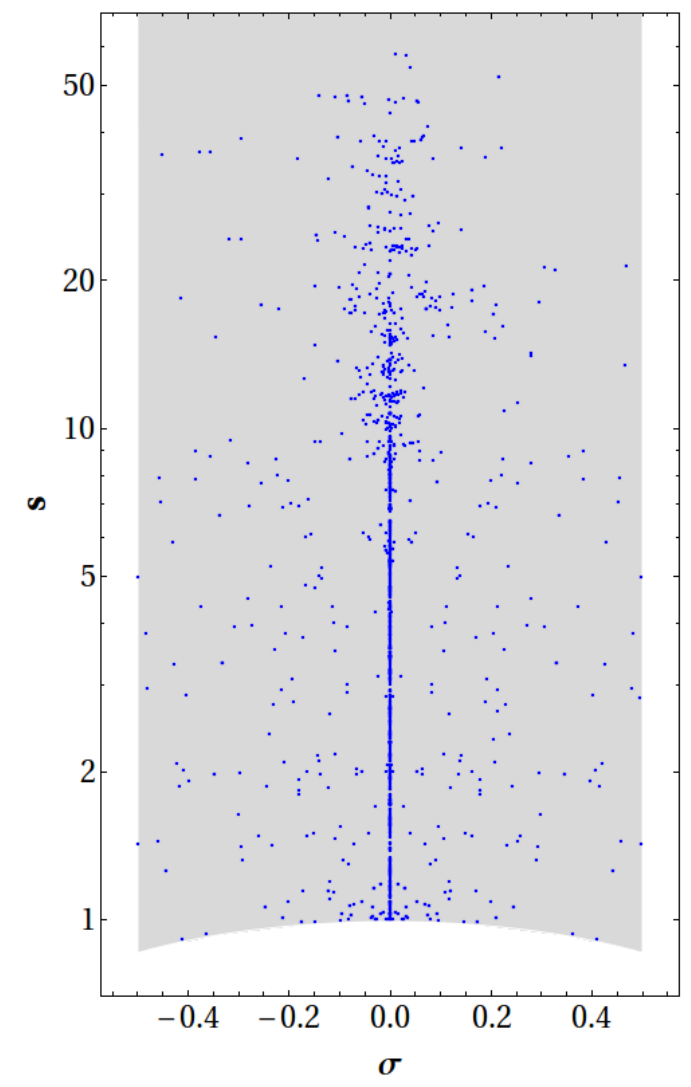

Figure 6. Distribution of $\tau$ for the minimal flux scan.
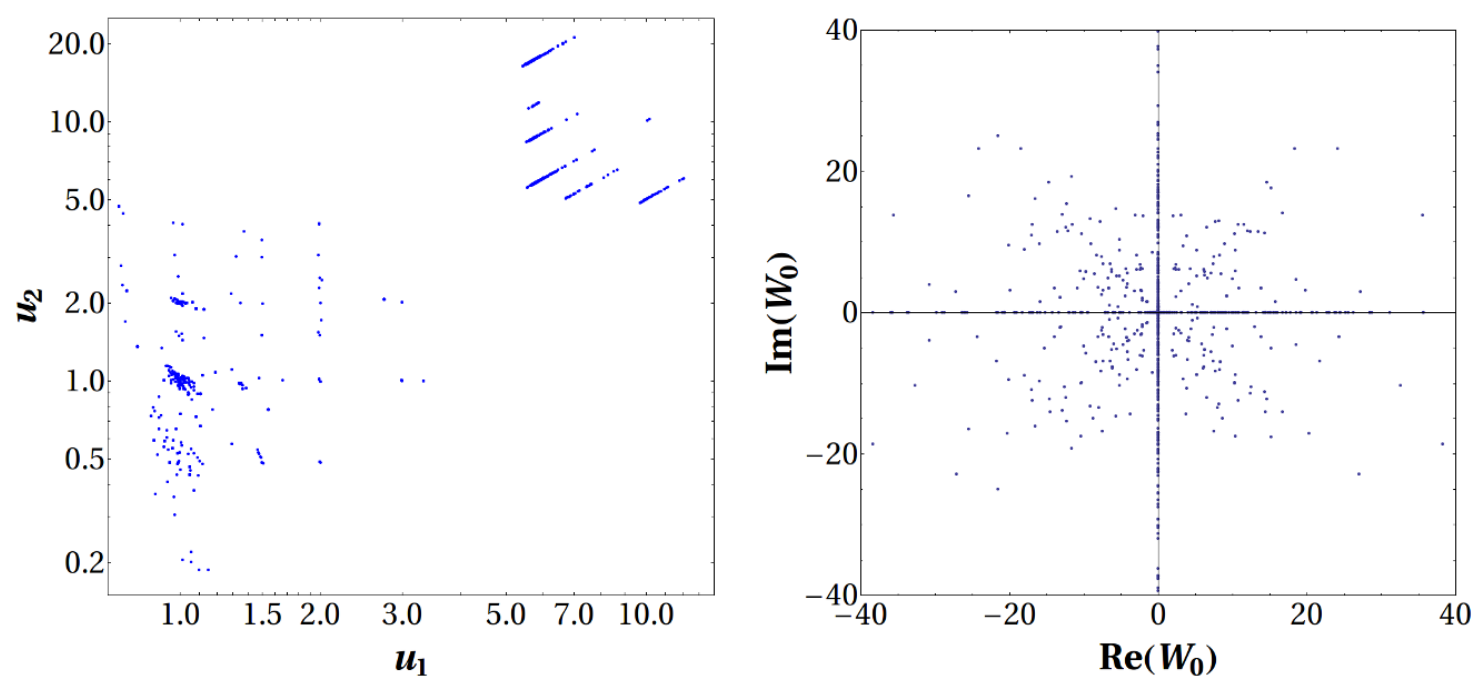

Figure 7. Distribution of $u_{1}$ and $u_{2}$ (left) and superpotential $W_{0}$ for the minimal flux scan.

around $W_{0}=0$, see figure 7 . This is however not a general property of the complete solution space as we noted in section 3.3 but rather $\mathcal{O}\left(10^{1}-10^{3}\right)$ values are preferred. 

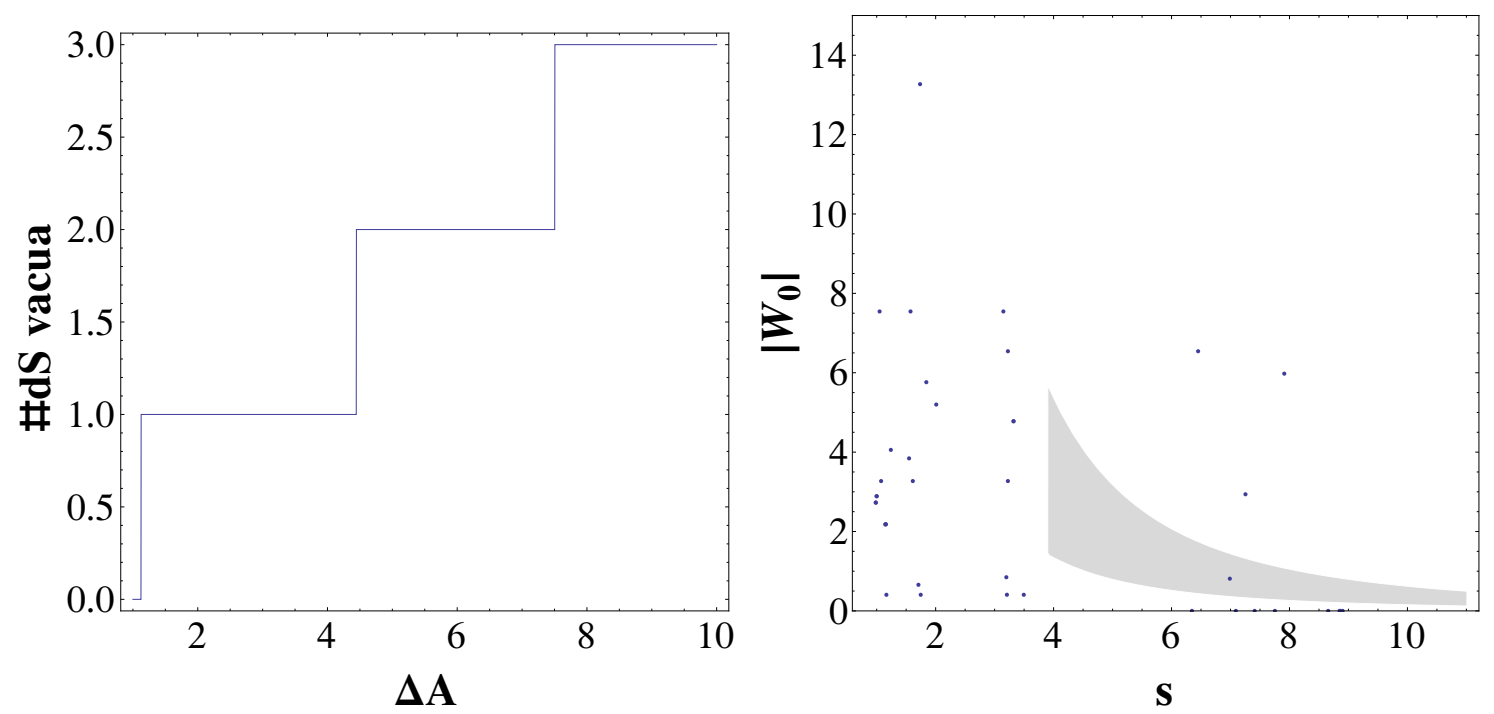

Figure 8. The number of Kähler uplifted dS vacua as a function of $\Delta A$ (left) and data points $\left(s,\left|W_{0}\right|\right)$ (right). Kähler uplifting can be applied in the shaded region $(\Delta A=2)$ for the vacua of the minimal flux scan with $L \leq 104$.

We fit the number of vacua as a function of the D3 tadpole $L$, finding $N_{\text {vac }} \simeq 0.02 L^{1.83}$ which strongly deviates from the estimate eq. (3.11). However, our dataset of 1,374 is in no way representative for the total number of flux vacua with $L=500$ such that this deviation can be easily explained by insufficient statistics.

Finally, we can calculate how many flux vacua allow a dS vacuum via Kähler uplifting along the lines of section 3.4. Since the minimal flux scan is setup such that the values of $s$ and $\left|W_{0}\right|$ naturally lie in the region where Kähler uplifting can be applied we find a much milder suppression of these vacua compared to section 3.3, see figure 8. For $\Delta A=10$, three of the 75 flux vacua with $L=104$ allow a dS vacuum via Kähler uplifting. Repeating the estimate of eq. (3.27) for the results of the minimal flux scan, the cosmological constant $\Lambda \sim 10^{-6}$ can be tuned to an accuracy

$$
\frac{\Delta \Lambda}{\Lambda} \sim 1.9 \pm 2.2
$$

To conclude this section, the minimal flux method has the advantage that one can specify the region in parameter space where physically interesting flux vacua should be constructed. In our case this region is defined by the large complex structure limit, weak string coupling and $\mathcal{O}(1)$ values of $W_{0}$. However, it is not possible to construct all flux vacua for a given D3 tadpole $L$ which can be done using paramotopy and the method is rather inefficient in the sense that only $0.01 \%$ of the starting values yield a flux vacuum.

\section{Conclusions}

In this paper we have studied the flux vacua of type IIB string theory compactified on the Calabi-Yau hypersurface $\mathbb{C P}_{1169}^{4}[18]$, i.e. the standard working example of both the 
LVS and the Kähler uplifting scenario. We switch on flux along six three-cycles that correspond to two complex structure moduli that are invariant under a certain discrete symmetry that can be used to construct the mirror manifold. As explained in the main text, such a supersymmetric vacuum in these two complex structure moduli extends to a supersymmetric vacuum of all 272 complex structure moduli.

To explicitly construct flux vacua, we make use of the fact that the prepotential $\mathcal{G}$ of the two complex structure moduli space has been worked out in the large complex structure limit. We apply two computational methods to find flux vacua on this manifold: the polynomial homotopy continuation method allows us to explicitly construct for the first time all flux vacua in the large complex structure limit that are consistent with a given D3 tadpole $L$ by applying the polynomial homotopy continuation method at each point in flux parameter space. The minimal flux method finds flux parameters that are consistent with a given D3 tadpole $L$ for a given set of vacuum expectation values (VEVs) of the complex structure moduli.

We analyze the resulting solution space of flux vacua for several physically interesting properties. For the polynomial homotopy continuation method, we find that for the $\sim$ 50,000 parameter points of our scan there are $\sim 20,000$ solutions in the large complex structure limit. We find a preference of strongly coupled vacua $g_{s} \gtrsim 1$ and preference for values of $\mathcal{O}\left(10^{1}-10^{3}\right)$ for the flux superpotential $W_{0}$. The number of vacua is

$$
N_{\mathrm{vac}} \simeq(0.50 \pm 0.04) L^{2.94 \pm 0.03},
$$

compared to $\sim 0.03 L^{3}$ expected form statistical analysis $[24,30]$. The gravitino mass is typically $m_{3 / 2}^{2}=\mathcal{O}\left(10^{-3}\right) \cdot\left(\frac{100}{\mathcal{V}}\right)^{2} M_{\mathrm{P}}^{2}$ and the masses of the complex structure moduli and the dilaton scale like $\mathcal{O}\left(10^{-3}-10^{2}\right) \cdot\left(\frac{100}{\mathcal{V}}\right)^{2} M_{\mathrm{P}}^{2}$. These ranges for the moduli and gravitino masses are compatible with the values obtained for a single explicit flux choice in the same construction $[14,15]$.

The average spacing of the flux superpotential in our solution set can be used to estimate the available fine-tuning $\Delta \Lambda / \Lambda$ of the cosmological constant $\Lambda$ as

$$
\frac{\Delta \Lambda}{\Lambda} \simeq(6.0 \pm 0.3) L^{-(0.95 \pm 0.005)\left(h_{\mathrm{eff}}^{2,1}+1\right)}
$$

which corresponds to for instance $\Delta \Lambda / \Lambda \sim 10^{-102}$ for $L=500$ and $h_{\text {eff }}^{2,1}=40$. The explicit brane and gauge flux construction in [15] allows us to answer the question how many of theses supersymmetric flux vacua allow an uplift to dS via Kähler uplifting. Depending on the available values for the one-loop determinant from gaugino condensation used to stabilize the Kähler moduli in this setup, we find that for a fraction of about $10^{-4}$ of all flux vacua up to a given D3-brane tadpole this mechanism can be applied to obtain a dS vacuum.

For the minimal flux method, we find $\sim 1000$ flux vacua with $L<500$ out of $\sim 10^{7}$ parameter points of our scan. This method allows us to control the region in $W_{0}$ and moduli space where we are intending to find flux vacua. Hence, we more easily access the regions of weak string coupling and the large complex structure limit compared to the polynomial homotopy continuation method. For the much smaller set of flux vacua constructed with the minimal flux method, the fraction of Kähler uplifted dS minima is about $10 \%$. 


\section{Acknowledgments}

We thank Frederik Denef for the initial motivation in launching this project, and Jose Blanco-Pillado, Arthur Hebecker, Jan Louis, Francisco Pedro, and Roberto Valandro for valuable, and enlightening discussions. MR thanks Malte Nuhn for help with Python. This work was supported by the Impuls und Vernetzungsfond of the Helmholtz Association of German Research Centers under grant HZ-NG-603, the German Science Foundation (DFG) within the Collaborative Research Center 676 "Particles, Strings and the Early Universe" and the Research Training Group 1670. DM would like to thank the U.S. Department of Energy for their support under contract no. DE-FG02-85ER40237; Fermilab's LQCD clusters where the cheater's homotopy runs were performed; and most importantly, Matthew Niemerg for his continuous support in installing and help in running Paramotopy. Finally, the authors would like to thank the organizers of the 2012 String Phenomenology conference in Cambridge where part of the work was done for their warm hospitality.

Open Access. This article is distributed under the terms of the Creative Commons Attribution License which permits any use, distribution and reproduction in any medium, provided the original author(s) and source are credited.

\section{References}

[1] Supernova Search Team collaboration, A.G. Riess et al., Observational evidence from supernovae for an accelerating universe and a cosmological constant, Astron. J. 116 (1998) 1009 [astro-ph/9805201] [INSPIRE].

[2] Supernova Cosmology Project collaboration, S. Perlmutter et al., Measurements of $\Omega$ and $\Lambda$ from 42 high redshift supernovae, Astrophys. J. 517 (1999) 565 [astro-ph/9812133] [INSPIRE].

[3] WMAP collaboration, E. Komatsu et al., Seven-year Wilkinson Microwave Anisotropy Probe (WMAP) observations: cosmological interpretation, Astrophys. J. Suppl. 192 (2011) 18 [arXiv: 1001.4538] [INSPIRE].

[4] K. Dasgupta, G. Rajesh and S. Sethi, M theory, orientifolds and G-flux, JHEP 08 (1999) 023 [hep-th/9908088] [INSPIRE].

[5] S.B. Giddings, S. Kachru and J. Polchinski, Hierarchies from fluxes in string compactifications, Phys. Rev. D 66 (2002) 106006 [hep-th/0105097] [INSPIRE].

[6] S. Kachru, R. Kallosh, A.D. Linde and S.P. Trivedi, De Sitter vacua in string theory, Phys. Rev. D 68 (2003) 046005 [hep-th/0301240] [INSPIRE].

[7] T.W. Grimm and J. Louis, The effective action of $N=1$ Calabi-Yau orientifolds, Nucl. Phys. B 699 (2004) 387 [hep-th/0403067] [INSPIRE].

[8] J.R. Ellis, A. Lahanas, D.V. Nanopoulos and K. Tamvakis, No-scale supersymmetric Standard Model, Phys. Lett. B 134 (1984) 429 [INSPIRE].

[9] E. Cremmer, S. Ferrara, C. Kounnas and D.V. Nanopoulos, Naturally vanishing cosmological constant in $N=1$ supergravity, Phys. Lett. B 133 (1983) 61 [INSPIRE].

[10] K. Becker, M. Becker, M. Haack and J. Louis, Supersymmetry breaking and $\alpha^{\prime}$ corrections to flux induced potentials, JHEP 06 (2002) 060 [hep-th/0204254] [INSPIRE]. 
[11] M. Berg, M. Haack and B. Körs, String loop corrections to Kähler potentials in orientifolds, JHEP 11 (2005) 030 [hep-th/0508043] [INSPIRE].

[12] V. Balasubramanian and P. Berglund, Stringy corrections to Kähler potentials, SUSY breaking and the cosmological constant problem, JHEP 11 (2004) 085 [hep-th/0408054] [INSPIRE].

[13] V. Balasubramanian, P. Berglund, J.P. Conlon and F. Quevedo, Systematics of moduli stabilisation in Calabi-Yau flux compactifications, JHEP 03 (2005) 007 [hep-th/0502058] [INSPIRE].

[14] M. Rummel and A. Westphal, A sufficient condition for de Sitter vacua in type IIB string theory, JHEP 01 (2012) 020 [arXiv:1107.2115] [inSPIRE].

[15] J. Louis, M. Rummel, R. Valandro and A. Westphal, Building an explicit de Sitter, JHEP 10 (2012) 163 [arXiv: 1208.3208] [INSPIRE].

[16] C. Burgess, R. Kallosh and F. Quevedo, De Sitter string vacua from supersymmetric D terms, JHEP 10 (2003) 056 [hep-th/0309187] [INSPIRE].

[17] M. Haack, D. Krefl, D. Lüst, A. Van Proeyen and M. Zagermann, Gaugino condensates and D-terms from D7-branes, JHEP 01 (2007) 078 [hep-th/0609211] [INSPIRE].

[18] M. Cicoli, S. Krippendorf, C. Mayrhofer, F. Quevedo and R. Valandro, D-branes at del Pezzo singularities: global embedding and moduli stabilisation, JHEP 09 (2012) 019 [arXiv:1206.5237] [INSPIRE].

[19] O. Lebedev, H.P. Nilles and M. Ratz, De Sitter vacua from matter superpotentials, Phys. Lett. B 636 (2006) 126 [hep-th/0603047] [INSPIRE].

[20] K.A. Intriligator, N. Seiberg and D. Shih, Dynamical SUSY breaking in meta-stable vacua, JHEP 04 (2006) 021 [hep-th/0602239] [INSPIRE].

[21] M. Cicoli, A. Maharana, F. Quevedo and C. Burgess, De Sitter string vacua from dilaton-dependent non-perturbative effects, JHEP 06 (2012) 011 [arXiv:1203.1750] [INSPIRE].

[22] R. Bousso and J. Polchinski, Quantization of four form fluxes and dynamical neutralization of the cosmological constant, JHEP 06 (2000) 006 [hep-th/0004134] [INSPIRE].

[23] J.L. Feng, J. March-Russell, S. Sethi and F. Wilczek, Saltatory relaxation of the cosmological constant, Nucl. Phys. B 602 (2001) 307 [hep-th/0005276] [INSPIRE].

[24] F. Denef and M.R. Douglas, Distributions of flux vacua, JHEP 05 (2004) 072 [hep-th/0404116] [INSPIRE].

[25] P. Candelas, A. Font, S.H. Katz and D.R. Morrison, Mirror symmetry for two parameter models. 2, Nucl. Phys. B 429 (1994) 626 [hep-th/9403187] [INSPIRE].

[26] B.R. Greene and M. Plesser, Duality in Calabi-Yau moduli space, Nucl. Phys. B 338 (1990) 15 [INSPIRE].

[27] O. DeWolfe, A. Giryavets, S. Kachru and W. Taylor, Type IIA moduli stabilization, JHEP 07 (2005) 066 [hep-th/0505160] [INSPIRE].

[28] F. Denef, M.R. Douglas and B. Florea, Building a better racetrack, JHEP 06 (2004) 034 [hep-th/0404257] [INSPIRE].

[29] A.J. Sommese and C.W. Wampler, The numerical solution of systems of polynomials arising in engineering and science, World Scientific Publishing Company, Singapore (2005). 
[30] S. Ashok and M.R. Douglas, Counting flux vacua, JHEP 01 (2004) 060 [hep-th/0307049] [INSPIRE].

[31] J.P. Conlon and F. Quevedo, On the explicit construction and statistics of Calabi-Yau flux vacua, JHEP 10 (2004) 039 [hep-th/0409215] [INSPIRE].

[32] A.D. Linde and A. Westphal, Accidental inflation in string theory, JCAP 03 (2008) 005 [arXiv:0712.1610] [INSPIRE].

[33] J.J. Blanco-Pillado, M. Gomez-Reino and K. Metallinos, Accidental inflation in the landscape, JCAP 02 (2013) 034 [arXiv:1209.0796] [INSPIRE].

[34] D. Marsh, L. McAllister and T. Wrase, The wasteland of random supergravities, JHEP 03 (2012) 102 [arXiv:1112.3034] [INSPIRE].

[35] X. Chen, G. Shiu, Y. Sumitomo and S.-H. Henry Tye, A global view on the search for de Sitter vacua in (type IIA) string theory, JHEP 04 (2012) 026 [arXiv:1112.3338] [INSPIRE].

[36] T.C. Bachlechner, D. Marsh, L. McAllister and T. Wrase, Supersymmetric vacua in random supergravity, JHEP 01 (2013) 136 [arXiv:1207.2763] [INSPIRE].

[37] Y. Sumitomo and S.-H. Henry Tye, A stringy mechanism for a small cosmological constant, JCAP 08 (2012) 032 [arXiv: 1204.5177] [INSPIRE].

[38] Y. Sumitomo and S.-H. Henry Tye, A stringy mechanism for a small cosmological constant - multi-moduli cases, JCAP 02 (2013) 006 [arXiv:1209.5086] [INSPIRE].

[39] Y. Sumitomo and S.-H. Henry Tye, Preference for a vanishingly small cosmological constant in supersymmetric vacua in a type IIB string theory model, Phys. Lett. B 723 (2013) 406 [arXiv: 1211.6858] [INSPIRE].

[40] M.R. Douglas and S. Kachru, Flux compactification, Rev. Mod. Phys. 79 (2007) 733 [hep-th/0610102] [INSPIRE].

[41] M. Graña, Flux compactifications in string theory: a comprehensive review, Phys. Rept. 423 (2006) 91 [hep-th/0509003] [INSPIRE].

[42] R. Blumenhagen, B. Körs, D. Lüst and S. Stieberger, Four-dimensional string compactifications with D-branes, orientifolds and fluxes, Phys. Rept. 445 (2007) 1 [hep-th/0610327] [INSPIRE].

[43] S. Gukov, C. Vafa and E. Witten, CFT's from Calabi-Yau four folds, Nucl. Phys. B 584 (2000) 69 [Erratum ibid. B 608 (2001) 477] [hep-th/9906070] [INSPIRE].

[44] L.E. Ibanez and A.M. Uranga, String theory and particle physics: an introduction to string phenomenology, Cambridge University Press, Cambridge U.K. (2012).

[45] P. Candelas, X. de la Ossa and F. Rodriguez-Villegas, Calabi-Yau manifolds over finite fields. 1, hep-th/0012233 [INSPIRE].

[46] A. Giryavets, S. Kachru, P.K. Tripathy and S.P. Trivedi, Flux compactifications on Calabi-Yau threefolds, JHEP 04 (2004) 003 [hep-th/0312104] [INSPIRE].

[47] D. Mehta, Lattice vs. continuum: Landau gauge fixing and 't Hooft-Polyakov monopoles, Ph.D. thesis, University of Adelaide, Australasian Digital Theses Program, Australia (2009).

[48] D. Mehta, A. Sternbeck, L. von Smekal and A.G. Williams, Lattice Landau gauge and algebraic geometry, PoS (QCD-TNT09) 025 [arXiv: 0912.0450] [INSPIRE]. 
[49] D. Mehta, Finding all the stationary points of a potential energy landscape via numerical polynomial homotopy continuation method, Phys. Rev. E 84 (2011) 025702 [arXiv: 1104.5497] [INSPIRE].

[50] D. Mehta, Numerical polynomial homotopy continuation method and string vacua, Adv. High Energy Phys. 2011 (2011) 263937 [arXiv:1108.1201] [InSPIRE].

[51] M. Kastner and D. Mehta, Phase transitions detached from stationary points of the energy landscape, Phys. Rev. Lett. 107 (2011) 160602 [arXiv:1108.2345] [INSPIRE].

[52] R. Nerattini, M. Kastner, D. Mehta and L. Casetti, Exploring the energy landscape of XY models, Phys. Rev. E 87 (2013) 032140 [arXiv:1211.4800] [INSPIRE].

[53] M. Maniatis and D. Mehta, Minimizing Higgs potentials via numerical polynomial homotopy continuation, Eur. Phys. J. Plus 127 (2012) 91 [arXiv:1203.0409] [INSPIRE].

[54] D. Mehta, Y.-H. He and J.D. Hauenstein, Numerical algebraic geometry: a new perspective on string and gauge theories, JHEP 07 (2012) 018 [arXiv:1203.4235] [INSPIRE].

[55] C. Hughes, D. Mehta and J.-I. Skullerud, Enumerating Gribov copies on the lattice, Annals Phys. 331 (2013) 188 [arXiv: 1203.4847] [INSPIRE].

[56] D. Mehta, J.D. Hauenstein and M. Kastner, Energy landscape analysis of the two-dimensional nearest-neighbor $\phi^{4}$ model, Phys. Rev. E 85 (2012) 061103 [arXiv:1202.3320] [INSPIRE].

[57] J. Hauenstein, Y.-H. He and D. Mehta, Numerical analyses on moduli space of vacua, arXiv:1210.6038 [INSPIRE].

[58] T.Y. Li, Solving polynomial systems by the homotopy continuation method, Handbook of numerical analysis XI (2003) 209.

[59] D.J. Bates, J.D. Hauenstein, A.J. Sommese and C.W. Wampler, Bertini: software for numerical algebraic geometry, available at http://www.nd.edu/ sommese/bertini.

[60] J. Verschelde, Algorithm 795: Phcpack: a general-purpose solver for polynomial systems by homotopy continuation, ACM Trans. Math. Soft. 25 (1999) 251.

[61] T. Gunji, S. Kim, M. Kojima, A. Takeda, K. Fujisawa and T. Mizutani, Phom: a polyhedral homotopy continuation method for polynomial systems, Computing 73 (2004) 57.

[62] T. Gao, T.Y. Li and M. Wu, Algorithm 846: Mixedvol: a software package for mixed-volume computation, ACM Trans. Math. Soft. 31 (2005) 555.

[63] D.N. Bernstein, The number of roots of a system of equations, Funct. Anal. Appl. 9 (1975) 183 [Funkts. Anal. Pril. 9 (1975) 1].

[64] A.G. Khovanski, Newton polyhedra and the genus of complete intersections, Funct. Anal. Appl. 12 (1978) 38 [Funkts. Anal. Pril. 12 (1978) 51.

[65] A.G. Kushnirenko, Newton polytopes and the Bezout theorem, Funct. Anal. Appl. 10 (1976) 233 [Funkts. Anal. Pril. 10 (1976) 82].

[66] T. Li, T. Sauer and J. Yorke, The cheater's homotopy: an efficient procedure for solving systems of polynomial equations, SIAM J. Numer. Anal. 26 (1989) 1241.

[67] T. Li and X. Wang, Nonlinear homotopies for solving deficient polynomial systems with parameters, SIAM J. Numer. Anal. 29 (1992) 1104.

[68] D.J. Bates, D.A. Brake and M.E. Niemerg., Efficient software for large-scale parameter homotopy problems, in preparation, (2012). 
[69] Y.-H. He, D. Mehta, M. Niemerg, M. Rummel and A. Valeanu, Exploring the potential energy landscape over a large parameter-space, arXiv:1301.0946 [INSPIRE].

[70] A. Westphal, De Sitter string vacua from Kähler uplifting, JHEP 03 (2007) 102 [hep-th/0611332] [INSPIRE].

[71] H. Cohen, A course in computational algebraic number theory, Springer, Germany (1993). 